\title{
ELECTRON TRANSPORT IN SEMICONDUCTOR SUPERLATTICES
}

\author{
BY
}

N. BEN ABDALLAH (Mathématiques pour l'Industrie et la Physique, UMR CNRS 5640, Université Paul Sabatier, 118, route de Narbonne, 31062 Toulouse cedex, France),

P. DEGOND (Mathématiques pour l'Industrie et la Physique, UMR CNRS 5640, Université Paul Sabatier, 118, route de Narbonne, 31062 Toulouse cedex, France),

A. MELLET (Mathématiques pour l'Industrie et la Physique, UMR CNRS 5640, Université Paul Sabatier, 118, route de Narbonne, 31062 Toulouse cedex, France and École Normale Supérieure de Lyon, 46, allée d'Italie, 69364 Lyon cedex 07, France),

AND

F. POUPAUD (Laboratoire de Mathématiques J. A. Dieudonné, UMR CNRS, Université de Nice Sophia Antipolis, Avenue de Valrose, 06108 Nice cedex 02, France)

\begin{abstract}
In this paper, we rigorously derive a diffusion model for semiconductor superlattices, starting from a kinetic description of electron transport at the microscopic scale. Electron transport in the superlattice is modelled by a collisionless Boltzmann equation subject to a periodic array of localized scatters modeling the periodic heterogeneities of the material. The limit of a large number of periodicity cells combined with a large-time asymptotics leads to a homogenized diffusion equation which belongs to the class of so-called "SHE" models (for Spherical Harmonics Expansion). The rigorous convergence proof relies on fine estimates on the operator modeling the localized scatters.
\end{abstract}

1. Introduction. The purpose of this paper is the rigorous derivation of a diffusion model for semiconductor superlattices, starting from a kinetic description of electron transport at the microscopic scale.

Semiconductor superlattices are processed by growing periodic layers of two different semiconductor materials, like $G a A s$ and $G a A l A s$ [25], [34]. The different electronic

Received January 5, 2001.

2000 Mathematics Subject Classification. Primary 35Q20, 76P05, 82A70, 78A35, 41A60.

Key words and phrases. Boltzmann equation, diffusion equation, spherical harmonics expansion, semiconductor superlattices, diffusion approximation, homogenization, interface operators, semigroups.

This work has been supported by the TMR network No. ERB FMBX CT97 0157 on "Asymptotic methods in kinetic theory" of the European Community and by the network "SPARCH" of the Centre National de la Recherche Scientifique.

E-mail address: naoufel@mip.ups-tlse.fr

E-mail address: degond@mip.ups-tlse.fr

E-mail address: mellet@mip.ups-tlse.fr

E-mail address: poupaud@math.unice.fr 
affinities of the two materials produce a periodic electrostatic potential in the direction of the growth axis, which is discontinuous at each interface between the two materials. Superlattices possess a number of interesting physical properties, especially regarding optoelectronics applications [25]. The modeling of electron transport in superlattices relies on efficient, yet phenomenological models, mostly based on Esaki and Tsu's model $[18]$.

Electron motion across the superlattice structure obeys quantum mechanics over length scales which are at least of the order of the superlattice period. However, the interaction of the electrons against various kinds of defects (such as the possible roughness of the material interfaces) can cause a breakdown of the electron phase coherences on larger length scales and thus destroy the quantum nature of the transport. In such a situation, Esaki and Tsu's model needs to be adapted. The model derived in the present paper is an attempt in this direction.

We consider a situation such that, within one superlattice period, one of the materials (denoted by $(N)$ ) is much narrower than the other one (denoted by $(W)$ ). We can conveniently describe the narrow material as a single plane at which the potential possesses a singularity and which acts as a localized source of scattering. The superlattice structure is therefore reduced to a periodic array of cells consisting of material $(W)$ separated by interfaces consisting of potential singularities. Electron motion within each cell is modeled by a collisionless Boltzmann equation for the distribution function (or the particle density in phase space (position, momentum)). The distribution functions in neighbouring cells are connected by means of a transmission operator, which describes the quantum scattering of the particles by the potential singularity. In this sense, the model retains the quantum nature of transport through the sharp potential inhomogeneities, while staying classical on larger scales.

The aim of this paper is to rigorously investigate the limit of a large number of cells and of large time. We suppose that there are $\alpha^{-1}$ superlattice cells within a macroscopic distance of the order of unity, while a given particle crosses $\alpha^{-2}$ cells within one unit of time, where $\alpha$ is a small parameter, typically the ratio of the superlattice period to the typical size of the device. Because the interface operators conserve particle energy, the limit model is a diffusion equation in an "augmented space" (position, energy), which is known as the "SHE" model (for Spherical Harmonics Expansion, a terminology arising from its early derivation by physicists, [8], [12] and references therein).

Homogenization limits of kinetic equations in the diffusion regime (diffusion approximation) have been widely investigated in the literature. Early formal approaches go back to [26], [27] for neutron transport while the mathematical theory was set up in [9]. The solution is formally expanded in powers of the small parameter $\alpha$. The problem is then formally reduced to a sequence of "cell problems" posed on the elementary period. Beyond homogenization problems, this expansion, which goes back to Hilbert and Chapman-Enskog, has been extensively used in diffusion limits of kinetic equations (see [28], [7] in neutron transport, [6] in radiative transfer and [31], [23], [8] in semiconductor physics). However, the reduction to a sequence of cell problems is not easily justified. To overcome this problem, the theory of two-scale convergence [29], [2] has recently been 
applied to the homogenization of kinetic equations in the diffusive regime [3], [24], [17] (see also [20], [1]).

The present paper is, to our knowledge, the first to deal with the homogenization of a kinetic problem in which the diffusion mechanism (the scattering by the interfaces) is concentrated at the boundary of the periodicity cells. We show that, in this particular situation, the diffusion limit can be rigorously justified without resorting to a cell problem nor to the theory of two-scale convergence. Besides, most of the previous works are concerned with diffusion models in position space only. As already pointed out, our limit regime is described by a diffusion equation posed on the extended position-energy space. Significant technical difficulties arise in the control of the additional energy variable. A derivation of the "SHE" model in the context of diffusion by the boundary has already been given in [14] (see also [5] and [13] for earlier work on these kinds of problems). However, in [14], the geometrical configuration of the electric field relative to the surface scattering operator makes the control of the energy variable less technical.

The "SHE" model has proven very useful in semiconductor physics (see, e.g., [33], [22]). It has first been derived in the case of standard semiconductor materials by [16] for the particular case of relaxation collision operators, and later extended to all elastic collision operators in [8] (see also [12] and references therein). Finally, in [15], an alternate SHE model for superlattices is formally derived. It relies on a discrete formulation of the original kinetic problem and applies to a broader class of superlattices.

The outline of the paper is as follows. In Sec. 2, the Boltzmann equation for superlattices, which is the starting point of our analysis, is introduced and appropriately scaled. The main theorem of the paper, i.e., the convergence of the Boltzmann equation towards the "SHE" model is also stated. In Sec. 3, the existence theory for the Boltzmann equation is given. First, properties of the energy band diagram (the energy versus momentum relationship) and of the interface operators are given. From this, the appropriate functional setting is defined. One of the key estimates, which gives the control of the trace of the distribution function on the interfaces in terms of the transport operator within the cells, is developed. It allows us to prove that the Boltzmann equation supplemented with the interface conditions admits solutions in an $L^{2}$ setting. The uniqueness of the solutions is left open in this work.

In Sec. 4, the proof of the main theorem, i.e., the convergence towards the SHE model, is developed. A first technical point is to prove that the weak $L^{2}$ limit of the distribution function depends on the energy only, instead of all components of momentum. The idea is to first prove this property for the traces on the interfaces, using the dissipative properties of the interface operator and then to "propagate" this property inside the cells. The second technical point is to prove that the current actually converges and to compute the limit. Again, the trace of the current at the interfaces is easy to compute by means of an auxiliary function that is a solution of a local problem (and not a cell problem). The point is again to prove that this property propagates inside the cells. The proofs of these two points rely on the estimate of the trace of the distribution function on the interfaces proved in Sec. 3 . 
2. The superlattice model and the scaling. At the microscopic scale, the unit length is chosen such that the superlattice period is 1 . Therefore, the $(N)$ interfaces are located at the points $x_{n}=n$ with $n \in \mathbb{Z}$. Let $f(x, k, t)$ be the electron distribution function in material $(W)$. The position variable $x$ is one dimensional and belongs to the real line except the location of the $(N)$ interfaces; so, $x \in \mathbb{R} \backslash \mathbb{Z}$. The momentum variable $k$ belongs to the first Brillouin zone $B$ associated with material $(W)$. The Brillouin zone is the fundamental domain of the torus $\mathbb{R}^{3} / L^{*}$, where $L^{*}$ is the reciprocal lattice of material $(W)$ (isomorphic to $\mathbb{Z}^{3}$ ). All functions of $k$ will be considered as periodic with periodicity $L^{*}$. Note that the $k$ variable is three dimensional even though the position variable $x$ is one dimensional. Finally, the time variable $t$ belongs to $[0, \infty)$.

The collisionless Boltzmann equation in material $(W)$ is written:

$$
\partial_{t} f+v_{x} \partial_{x} f+\partial_{x} V \partial_{k_{x}} f=0, \quad x \in \mathbb{R} \backslash \mathbb{Z}, k \in B, t \geq 0 .
$$

Here, $v_{x}=v_{x}(k)$ is the $x$-component of the particle velocity $v(k)$ defined by

$$
v(k)=\nabla_{k} \varepsilon(k), \quad k \in B,
$$

where $\varepsilon(k)$ is a given smooth periodic function of $k$ that gives the energy-wave-vector relationship in material $(W)$ (the so-called band diagram). $V=V(x)$ is the electrostatic potential due to charges and externally applied biases. It will be assumed given, time independent and as regular as necessary.

For a function $\varphi(x, k)$ defined on $\mathbb{R} \backslash \mathbb{Z}$, we denote by $\gamma_{n}^{ \pm}(\varphi)$ the following limits (if they exist):

$$
\gamma_{n}^{ \pm}(\varphi)(k)=\lim _{x \rightarrow n \pm 0} \varphi(x, k)
$$

and we define the outgoing and incoming traces of $\varphi$ at point $n$ respectively by:

$$
\begin{aligned}
& \gamma_{n}^{\text {out }}(\varphi)(k)= \begin{cases}\gamma_{n}^{-}(\varphi), & v_{x}(k)>0, \\
\gamma_{n}^{+}(\varphi), & v_{x}(k)<0,\end{cases} \\
& \gamma_{n}^{\text {inc }}(\varphi)(k)= \begin{cases}\gamma_{n}^{+}(\varphi), & v_{x}(k)>0, \\
\gamma_{n}^{-}(\varphi), & v_{x}(k)<0 .\end{cases}
\end{aligned}
$$

The outgoing (respectively incoming) trace is the distribution of particles leaving (respectively entering) the $(W)$ material at the $(N)$ interface located at $n$.

We suppose that the traces of $f$ are defined and that the $W-N-W$ structure can be conveniently described by a scattering operator that maps the outgoing trace at each interface to the corresponding incoming trace:

$$
\gamma_{n}^{\text {inc }}(f)=\mathcal{B}_{n}\left(\gamma_{n}^{\text {out }}(f)\right)
$$

This scattering operator $\mathcal{B}_{n}$ is an integral operator given for any function $\varphi(k)$ defined on $B$ by

$$
\mathcal{B}_{n} \varphi(k)=\int_{B} \sigma_{n}\left(k^{\prime}, k\right) \varphi\left(k^{\prime}\right)\left|v_{x}(k)^{\prime}\right| \delta\left(\varepsilon\left(k^{\prime}\right)-\varepsilon(k)\right) d k^{\prime} .
$$

The scattering cross section $\sigma_{n}$ can be derived from the quantum scattering analysis of the potential profile associated with the $W-N-W$ structure. Precisely, if $v_{x}\left(k^{\prime}\right)>0$ and $v_{x}(k)>0,\left|v_{x}(k)\right| \sigma_{n}\left(k^{\prime}, k\right) \delta\left(\varepsilon\left(k^{\prime}\right)-\varepsilon(k)\right) d k$ is the number of electrons transmitted through 
the interface to the right into a volume $d k$ around $k$ for one incident electron on the left with wave vector $k^{\prime}$. Similarly, if $v_{x}\left(k^{\prime}\right)>0$ and $v_{x}(k)<0,\left|v_{x}(k)\right| \sigma_{n}\left(k^{\prime}, k\right) \delta\left(\varepsilon\left(k^{\prime}\right)-\right.$ $\varepsilon(k)) d k$ is the number of electrons reflected by the interface to the left into a volume $d k$ around $k$ for one incident electron on the left with wave vector $k^{\prime}$. Similar interpretations are valid respectively for $v_{x}(k)<0, v_{x}\left(k^{\prime}\right)<0$ and $v_{x}(k)<0, v_{x}\left(k^{\prime}\right)>0$. $\sigma_{n}$ depends on $n$ to allow smooth changes (on a macroscopic scale) in the $W-N-W$ structure.

The passage to the macroscopic scale is done by the diffusion rescaling

$$
x^{\prime}=\alpha x, \quad t^{\prime}=\alpha^{2} t,
$$

where $\alpha \ll 1$ is a small parameter that is the ratio of the microscopic unit length (the superlattice period) to the macroscopic unit length (the typical size of the device). The square of $\alpha$ appears in the time rescaling because we are aiming at a diffusion model at the macroscopic scale. Setting

$$
f^{\alpha}\left(x^{\prime}, k, t^{\prime}\right)=f(x, k, t), \quad V\left(x^{\prime}\right)=V(x),
$$

we obtain the following model, which is the starting point of our analysis (where we have dropped the primes for clarity):

$$
\begin{aligned}
& \alpha \partial_{t} f^{\alpha}+v_{x}(k) \partial_{x} f^{\alpha}+\partial_{x} V \partial_{k_{x}} f^{\alpha}=0, \quad x \in \mathbb{R} \backslash \alpha \mathbb{Z}, k \in B, t \geq 0, \\
& \gamma_{n \alpha}^{\text {inc }}\left(f^{\alpha}\right)=\mathcal{B}_{\alpha n}\left(\gamma_{n \alpha}^{\text {out }}\left(f^{\alpha}\right)\right) .
\end{aligned}
$$

Note that the electrostatic potential is assumed independent of $\alpha$, which amounts to supposing that it varies over the macroscopic scale only. In this paper, we shall suppose

Hypothesis 2.1. The electric field $\partial_{x} V$ belongs to the Sobolev space $W^{1, \infty}$ (in other words, $\partial_{x} V$ is bounded and globally Lipschitz over $\mathbb{R}$ ).

Similarly, we suppose that there is a smooth function $\sigma\left(x, k^{\prime}, k\right)$ of the macroscopic variable $x$, such that

$$
\sigma_{n}\left(k^{\prime}, k\right)=\sigma\left(n \alpha, k^{\prime}, k\right),
$$

and we define the operator $\mathcal{B}(x)$ by

$$
\mathcal{B}(x) \varphi(k)=\int_{B} \sigma\left(x, k^{\prime}, k\right) \varphi\left(k^{\prime}\right)\left|v_{x}\left(k^{\prime}\right)\right| \delta\left(\varepsilon\left(k^{\prime}\right)-\varepsilon(k)\right) d k^{\prime} .
$$

Then, the interface condition (2.7) is also written

$$
\gamma_{n \alpha}^{\text {inc }}\left(f^{\alpha}\right)=\mathcal{B}(n \alpha)\left(\gamma_{n \alpha}^{\text {out }}\left(f^{\alpha}\right)\right)
$$

In this paper, we are concerned with the limit $\alpha \rightarrow 0$ of the kinetic model (2.6) and (2.9), with initial data

$$
f^{\alpha}(x, k, t=0)=f_{I}^{\alpha}(x, k), \quad x \in \mathbb{R} \backslash \alpha \mathbb{Z}, k \in B,
$$

also satisfying the transmission conditions (2.9). To avoid the treatment of initial layers, we shall suppose

Hypothesis 2.2. There exists a smooth function $F_{I}(x, \varepsilon)$ defined on $\mathbb{R} \times \mathcal{R}$, where $\mathcal{R}$ is the closure of the numerical range of the function $\varepsilon(k)$, satisfying

$$
F_{I}(x, \varepsilon(k)) \in L^{2}(\mathbb{R} \times B), \quad\left(v_{x} \partial_{x}+\partial_{x} V \partial k_{x}\right)\left[F_{I}(x, \varepsilon(k))\right] \in L^{2}(\mathbb{R} \times B),
$$

and such that

$$
f_{I}^{\alpha}(x, k)=\left.F_{I}(x, \varepsilon(k))\right|_{\mathbb{R} \backslash \alpha \mathbb{Z} \times B} .
$$


In other words, $f_{I}^{\alpha}$ is the restriction to $\mathbb{R} \backslash \alpha \mathbb{Z} \times B$ of an everywhere defined function independent of $\alpha$. Since $F_{I}$ depends on $k$ through the energy $\varepsilon(k)$ only and satisfies the regularity assumption (2.11), the initial datum (2.12) satisfies the interface condition (2.9) (see Sec. 3). In this paper, we are concerned with proving the following result:

Theorem 2.1. (i) Under the hypotheses listed in the current and following sections (namely Hypotheses 2.1, 2.2, 3.1, 3.2, 3.3, 3.4), the problem (2.6), (2.7) has a solution $f^{\alpha}$ (in a sense that will be specified further, see proposition 3.11 ).

(ii) When $\alpha$ tends to zero, $f^{\alpha}$ converges to $f^{0}$ in the weak star topology of $L^{\infty}([0, T]$, $\left.L^{2}(\mathbb{R} \times B)\right)$ for any $T>0$, where $f^{0}(x, k, t)=F(x, \varepsilon(k), t)$ and $F(x, \varepsilon, t)$ is the weak solution of the problem (SHE model) posed on the domain $(x, \varepsilon) \in \mathbb{R} \times \mathcal{R}$ :

$$
\begin{aligned}
& N(\varepsilon) \partial_{t} F+\left(\partial_{x}+\partial_{x} V \partial_{\varepsilon}\right) J=0, \\
& J(x, \varepsilon, t)=-D(x, \varepsilon)\left(\partial_{x}+\partial_{x} V \partial_{\varepsilon}\right) F . \\
& F(x, \varepsilon, t=0)=F_{I}(x, \varepsilon), \\
& J(x, \varepsilon, t)=0, \quad \varepsilon \in \partial \mathcal{R} .
\end{aligned}
$$

$N(\varepsilon)$ is the density-of-states of material $(W)$, whose definition is given in (3.2). The diffusion constant $D(x, \varepsilon)$ is given by

$$
D(x, \varepsilon)=\int_{B} v_{x}(k) \chi(x, k) \delta(\varepsilon(k)-\varepsilon) d k,
$$

where $\chi(x, k)$ is uniquely determined by the problem

$$
\begin{gathered}
\left(I-\mathcal{B}(x)^{*}\right)(\chi)=\frac{1}{2}\left(I+\mathcal{B}(x)^{*}\right)\left(\operatorname{sgn}\left(v_{x}\right)\right), \\
\int_{B} \chi(x, k)\left|v_{x}(k)\right| \delta(\varepsilon(k)-\varepsilon) d k=0, \quad \forall(x, \varepsilon) \in \mathbb{R} \times \mathcal{R},
\end{gathered}
$$

and where $\mathcal{B}^{*}$ is the adjoint of the operator $\mathcal{B}$. The diffusion constant $D(x, \varepsilon)$ is strictly positive for $(x, \varepsilon) \in \mathbb{R} \times \stackrel{\circ}{\mathcal{R}}$, where $\stackrel{\circ}{\mathcal{R}}$ denotes the interior of $\mathcal{R}$.

Let us note that the uniqueness of the solution of $(2.6),(2.7)$ is an open problem. This question is discussed in detail in the next section. The positivity of the diffusion constant $D$ guarantees that problem (2.13)-(2.16) is well-posed. The diffusion constant is the same as in [15] (see formula (4.43)), but the density-of-states $N(\varepsilon)$ is different, due to some differences in the original kinetic model. In the next section, we introduce the functional setting, and prove the existence and uniqueness of the solution. 


\section{Existence of the solution.}

3.1. Notation. We denote by $\Omega^{\alpha}=\mathbb{R} \backslash \alpha \mathbb{Z}, \mathcal{O}^{\alpha}=\Omega^{\alpha} \times B$, and $\Gamma^{\alpha}=\alpha \mathbb{Z} \times B$. $\mathcal{O}^{\alpha}$ is equipped with the usual $L^{2}$ norm and inner product. Note that $L^{2}\left(\mathcal{O}^{\alpha}\right)=L^{2}(\mathbb{R} \times B)$ since $\alpha \mathbb{Z} \times B$ is a zero measure set. Then

$$
|u|_{L^{2}\left(\mathcal{O}^{\alpha}\right)}^{2}=\int_{\mathbb{R} \times B}|u(x, k)|^{2} d x d k .
$$

We denote by $L_{\delta}^{2}(B)$ the weighted $L^{2}$ space, equipped with norm

$$
|u|_{L_{\delta}^{2}(B)}^{2}=\int_{B}|u(k)|^{2} w^{\delta}(k) d k
$$

with $w^{\delta}(k)=v_{x}(k) s^{\delta}\left(v_{x}(k)\right)$, where $s^{\delta}(x)$ is the continuous piecewise linear function equal to the sign function for $|x| \geq \delta$ :

$$
s^{\delta}(x)= \begin{cases}\operatorname{sgn}(x) & \forall|x| \geq \delta \\ \frac{x}{\delta} & \forall|x| \leq \delta .\end{cases}
$$

Let us remark that $L_{\delta}^{2}(B)$ is nothing but the weighted $L^{2}$ space associated with the weight $\left|v_{x}(k)\right|$, which will be denoted by $L^{2}(B)$.

3.2. Geometrical preliminaries. This section is devoted to some geometrical properties of the energy function $\varepsilon(k)$. We first suppose that $\varepsilon$ is a $C^{2}$ function on $B$ with values in $\mathbb{R}$, whose periodic extension to the whole space $\mathbb{R}^{3}$ is a $C^{2}$ function. For any energy $\varepsilon \in \mathbb{R}$, we denote by $\mathcal{S}_{\varepsilon}$ the constant energy surface $\mathcal{S}_{\varepsilon}=\{k \in B, \varepsilon(k)=\varepsilon\}$. Let $d S_{\varepsilon}(k)$ be the Euclidean surface element on $S_{\varepsilon}$ and $\mathcal{R}$ the closure of the numerical range of $\varepsilon(k)$. We also denote by $d N_{\varepsilon}(k)$ the co-area and by $N(\varepsilon)$ the energy-density of states:

$$
d N_{\varepsilon}(k)=\frac{d S_{\varepsilon}(k)}{\left|\nabla_{k} \varepsilon(k)\right|}, \quad N(\varepsilon)=\int_{\mathcal{S}_{\varepsilon}} d N_{\varepsilon}(k) .
$$

By Sard's theorem and the implicit function theorem, these objects are defined for all $\varepsilon \in \mathcal{R}_{0}$, where $\mathcal{R} \backslash \mathcal{R}_{0}$ is the set of critical values for the function $k \rightarrow \varepsilon(k)$ and is of measure zero.

For further simplifications, we suppose that $\mathcal{R} \backslash \mathcal{R}_{0}$ is a finite set, associated with a finite set $B \backslash \mathcal{B}_{0}$ of critical points. In order to use the Morse lemma, we also assume that these critical points are nondegenerate. Hence, for $k_{0} \in B \backslash B_{0}$, and $\varepsilon_{0}=\varepsilon\left(k_{0}\right)$, there exists a neighborhood $\mathcal{U}$ of $k_{0}$ and a diffeomorphism $\varphi$ such that, either $\varepsilon \circ \varphi=\varepsilon_{0}+k_{x}^{2}+k_{y}^{2}+k_{z}^{2}$ (critical point of parabolic type), or $\varepsilon \circ \varphi=\varphi_{0}+k_{x}^{2}+k_{y}^{2}-k_{z}^{2}$ (critical point of hyperbolic type). A simple computation gives in the first case

$$
\lim _{\varepsilon \rightarrow \varepsilon_{0}} \int_{\mathcal{S}_{\varepsilon} \cap \mathcal{U}} d N_{\varepsilon}(k) \rightarrow 0
$$

and in the second case

$$
\lim _{\varepsilon \rightarrow \varepsilon_{0}} \int_{\mathcal{S}_{\varepsilon} \cap \mathcal{U}} d N_{\varepsilon}(k) \rightarrow C<+\infty .
$$

It is now easy to check that $N(\varepsilon) \in L^{\infty}(\mathcal{R})$, since $N(\varepsilon)$ can be extended as a continuous function on the compact set $\mathcal{R}$. With this hypothesis, the co-area formula applies [19]: 
for any continuous function $\psi(k)$ defined on $B$, we have:

$$
\int_{B} \psi(k) d k=\int_{\mathcal{R}}\left(\int_{\mathcal{S}_{\varepsilon}} \psi(k) \frac{d S_{\varepsilon}(k)}{\left|\nabla_{k} \varepsilon(k)\right|}\right) d \varepsilon:=\int_{\mathcal{R}}\left(\int_{\mathcal{S}_{\varepsilon}} \psi(k) d N_{\varepsilon}(k)\right) d \varepsilon .
$$

For $\varepsilon \in \mathcal{R}$, we define $L_{\delta}^{2}\left(\mathcal{S}_{\varepsilon}\right)$ equipped with the norm

$$
|u(k)|_{L_{\delta}^{2}\left(\mathcal{S}_{\varepsilon}\right)}^{2}=\int_{\mathcal{S}}|u(k)|^{2}\left|w^{\delta}(k)\right| d N_{\varepsilon}(k),
$$

so that $L_{\delta}^{2}(B)=L^{2}\left(\mathcal{R}, L_{\delta}^{2}\left(\mathcal{S}_{\varepsilon}\right)\right)$ by the co-area formula. We denote by $L_{\delta}^{2}\left(\Gamma^{\alpha}\right)$ the set of functions $u=\left(u_{\alpha n}\right)_{n \in \mathbb{Z}}$, such that $u_{\alpha n} \in L_{\delta}^{2}(B)$ for all $n \in \mathbb{Z}$ and such that

$$
|u|_{L_{\delta}^{2}\left(\Gamma^{\alpha}\right)}^{2}:=\sum_{n \in \mathbb{Z}} \alpha\left|u_{\alpha n}\right|_{L_{\delta}^{2}(B)}^{2} .
$$

Similarly, we define $L^{2}\left(\mathcal{S}_{\varepsilon}\right), L^{2}\left(\Gamma^{\alpha}\right)$, associated with the weight $\left|v_{k}(k)\right|=w^{0}(k)$. We now assume another geometrical condition on the energy function $\varepsilon(k)$, which is always fulfilled in practice.

Hypothesis 3.1. For any $\varepsilon$ in the interior $\stackrel{\circ}{\mathcal{R}}$ of the range of $\mathcal{R}$ of $\varepsilon$, we have

$$
\int_{\mathcal{S}_{\varepsilon}}\left|v_{x}(k)\right| d N_{\varepsilon}(k)>0 .
$$

In other words, for all $\varepsilon \in \stackrel{\circ}{\mathcal{R}}$, we have $v_{x}(k) \neq 0$ on a nonnegligible set of $\mathcal{S}_{\varepsilon}$ for the measure $d N_{\varepsilon}(k)$. It is clear that the above hypothesis is automatically satisfied for energies $\varepsilon_{0} \in \stackrel{\circ}{\mathcal{R}} \backslash \mathcal{R}_{0}$. Indeed, it is readily seen that, for such energies, there exists $k \in \mathcal{S}_{\varepsilon_{0}}$ that is not a critical point of parabolic type (since such points are associated with local extrema of the function $\varepsilon$ ) and by a simple computation, we see that for any critical point of hyperbolic type, we have $\lim _{\varepsilon \rightarrow \varepsilon_{0}} \int_{\mathcal{S}_{\varepsilon} \cap \mathcal{U}}\left|v_{x}(k)\right| d N_{\varepsilon}(k) \neq 0$. We deduce the following lemma.

LEMma 3.1. For any compact set $K \subset \stackrel{\circ}{\mathcal{R}}$, and all $\delta_{0}>0$, there exists a constant $C_{K, \delta_{0}}>0$, such that, $\forall \delta, 0 \leq \delta \leq \delta_{0}, \forall \varepsilon \in K$, we have

$$
\int_{S_{\varepsilon}}\left|v_{x}(k)\right| d N_{\varepsilon}(k) \leq C_{K, \delta_{0}} \int_{\mathcal{S}_{\varepsilon}} w^{\delta}(k) d N_{\varepsilon}(k) .
$$

Proof. If the converse were true, there would exist a compact set $K_{0} \subset \stackrel{\circ}{\mathcal{R}}$, a real number $\delta_{0}>0$, and two sequences, $\varepsilon_{n} \in K_{0}, \delta_{n} \in\left[0, \delta_{0}\right]$, such that

$$
\int_{S_{\varepsilon_{n}}}\left|v_{x}(k)\right| d N_{\varepsilon_{n}}(k) \geq n \int_{\mathcal{S}_{\varepsilon_{n}}} w^{\delta_{n}}(k) d N_{\varepsilon_{n}}(k) .
$$

Then, since

$$
\int_{S_{\varepsilon_{n}}}\left|v_{x}(k)\right| d N_{\varepsilon_{n}}(k) \leq\left(\sup _{k \in B}\left|v_{x}(k)\right|\right) N\left(\varepsilon_{n}\right) \leq C
$$

we have

$$
\limsup _{n \rightarrow 0} \int_{\mathcal{S}_{\varepsilon_{n}}} w^{\delta_{n}}(k) d N_{\varepsilon_{n}}(k)=0 .
$$


Let $\varepsilon$ be a limit point of the sequence $\left(\varepsilon_{n}\right)$. Then $\varepsilon \in \stackrel{\circ}{\mathcal{R}}$ and the above properties imply that $\left|v_{x}(k)\right|=0$ almost everywhere on $\mathcal{S}_{\varepsilon}$ with respect to the measure $d N_{\varepsilon}(k)$, which is in contradiction with Hypothesis 3.1 .

3.3. Properties of the operator $\mathcal{B}$. We now list the required assumptions on the operator $\mathcal{B}(x)$.

Hypothesis 3.2. (i) Positivity: $\sigma>0$, almost everywhere.

(ii) Particle conservation:

$$
\int_{B} \sigma\left(x, k^{\prime}, k\right)\left|v_{x}(k)\right| \delta\left(\varepsilon\left(k^{\prime}\right)-\varepsilon(k)\right) d k=1, \quad \forall k^{\prime} \in B .
$$

(iii) Reciprocity:

$$
\sigma\left(x, k^{\prime}, k\right)=\sigma\left(x,-k,-k^{\prime}\right) .
$$

(iv) $\mathcal{B}(x)$ is compact on $L^{2}\left(\mathcal{S}_{\varepsilon}\right)$, for all $\varepsilon \in \mathcal{R}$, and $x \in \mathbb{R}$.

Property (i) expresses the obvious physical constraint that a number of particles must be positive (see the interpretation of $\sigma$ in Sec. 2). Property (ii) states that the flux of particles of given energy $\varepsilon$ is preserved by the transmission operator. It is true as long as there is no scattering between different energy states (via, e.g., phonons). An account of inelastic scattering in the present analysis is possible provided that it remains weak. This will be developed in a future work. Equation (3.5) can be rewritten with the co-area formula:

$$
\int_{\mathcal{S}_{\varepsilon\left(k^{\prime}\right)}} \sigma\left(x, k^{\prime}, k\right)\left|v_{x}(k)\right| d N_{\varepsilon\left(k^{\prime}\right)}(k)=1 .
$$

Property (iii) expresses the time reversibility of the microscopic scattering process. Combined with (3.5), Eq. (3.6) yields the following identity, further referred to as the normalization condition:

$$
\int_{B} \sigma\left(x, k^{\prime}, k\right)\left|v_{x}\left(k^{\prime}\right)\right| \delta\left(\varepsilon\left(k^{\prime}\right)-\varepsilon(k)\right) d k^{\prime}=1, \quad \forall k \in B,
$$

or

$$
\int_{\mathcal{S}_{\varepsilon(k)}} \sigma\left(x, k^{\prime}, k\right)\left|v_{x}\left(k^{\prime}\right)\right| d N_{\varepsilon(k)}\left(k^{\prime}\right)=1 .
$$

From (3.9) and the Cauchy-Schwarz inequality, we easily deduce the following inequality:

$$
|\mathcal{B}(x) u|_{L^{2}\left(\mathcal{S}_{\varepsilon}\right)}^{2} \leq|u|_{L^{2}\left(\mathcal{S}_{\varepsilon}\right)}^{2},
$$

which plays a similar role to the Darrozes-Guiraud inequality in gas-surface interaction [11]. Since by the normalization condition (3.9), constant functions are fixed points of $\mathcal{B}(x),(3.10)$ implies that for each $\varepsilon \in \mathcal{R}$, the operator $\mathcal{B}(x)$ is a continuous linear operator on $L^{2}\left(\mathcal{S}_{\varepsilon}\right)$ with norm 1 :

$$
\|\mathcal{B}(x)\|_{\mathcal{L}\left(L^{2}\left(\mathcal{S}_{\varepsilon}\right)\right)}=1, \quad \forall \varepsilon \in \mathcal{R} .
$$

We denote by $\mathcal{B}^{\alpha}$ the operator on $L^{2}\left(\Gamma^{\alpha}\right)$ that coincides with $\mathcal{B}(\alpha n)$ on each interface, namely:

$$
u=\left(u_{\alpha n}\right)_{n \in \mathbb{Z}} \in L^{2}\left(\Gamma^{\alpha}\right) \rightarrow \mathcal{B}^{\alpha} u=\left(\mathcal{B}(\alpha n) u_{\alpha n}\right)_{n \in \mathbb{Z}} \in L^{2}\left(\Gamma^{\alpha}\right) .
$$

Obviously, $\mathcal{B}^{\alpha}$ is a continuous operator on $L^{2}\left(\Gamma^{\alpha}\right)$. Moreover, as a consequence of hypothesis (i), $\mathcal{B}(x) u>0$ for any nonnegative and nonidentically vanishing function $u \in L^{2}\left(\mathcal{S}_{\varepsilon}\right)$. This implies the following proposition. 
Proposition 3.2. (i) For all $x \in \mathbb{R}$ and all $\varepsilon \in \mathcal{R}, \mathcal{B}(x)$ considered as an operator on $L^{2}\left(\mathcal{S}_{\varepsilon}\right)$ has 1 as an eigenvalue with multiplicity 1 . The associated eigenspace is the set of constant functions. The eigenvalue 1 is the only eigenvalue associated with a signed eigenfunction.

(ii) We have

$$
N(I-\mathcal{B}(x))=\mathbb{R}
$$

where $N$ denotes the Null-Space of an operator defined on $L^{2}\left(\mathcal{S}_{\varepsilon}\right)$.

Proof. (ii) is a direct consequence of (i). From the normalization condition (3.8), the constant functions are eigenfunctions of $\mathcal{B}(x)$ associated with the eigenvalue 1 . By Hypothesis 3.2, Krein-Rutman's theorem [10] applies and 1 is the only eigenvalue of $\mathcal{B}(x)$ and it is simple, which proves (i).

Let us now denote by $Q$ the orthogonal projector of $L^{2}\left(\mathcal{S}_{\varepsilon}\right)$ onto the space of constant functions, namely

$$
Q u(k)=\mathcal{V}(\varepsilon)^{-1} \int_{\mathcal{S}_{\varepsilon}} u(k)\left|v_{x}(k)\right| d N_{\varepsilon}(k), \quad \mathcal{V}(\varepsilon)=\int_{\mathcal{S}_{\varepsilon}}\left|v_{x}(k)\right| d N_{\varepsilon}(k),
$$

and introduce

$$
P=I-Q,
$$

where $I$ is the identity on $L^{2}\left(\mathcal{S}_{\varepsilon}\right)$. Note that the adjoint $\mathcal{B}^{*}(x)$ of $\mathcal{B}(x)$ is given by

$$
\mathcal{B}^{*}(x) u(k)=\int_{\mathcal{S}_{\varepsilon}} \sigma\left(x, k, k^{\prime}\right) u\left(k^{\prime}\right)\left|v_{x}\left(k^{\prime}\right)\right| d N_{\varepsilon(k)}\left(k^{\prime}\right)
$$

and satisfies, thanks to the flux condition (3.5), the following property:

$$
\mathbb{R} \subset N\left(I-\mathcal{B}^{*}(x)\right) .
$$

The following lemma will be needed in the sequel.

Lemma 3.3. The operators $P, Q$, and $\mathcal{B}(x)$ satisfy the following identities:

$$
P \mathcal{B}(x)=\mathcal{B}(x) P, \quad Q \mathcal{B}(x)=\mathcal{B}(x) Q=Q .
$$

Proof. Let $u$ be an arbitrary function of $L^{2}\left(\mathcal{S}_{\varepsilon}\right)$. We write, omitting the $x$-dependence of $\mathcal{B}$ :

$$
\mathcal{B} u=\mathcal{B} P u+\mathcal{B} Q u \text {. }
$$

Since $Q u$ is a constant function, $\mathcal{B} Q u=Q u$. Hence $\mathcal{B} Q=Q$. Moreover, (3.15) yields $\mathcal{B}^{*} Q=Q$. Taking the adjoint of this identity leads to $Q \mathcal{B}=Q$. The identity $\mathcal{B} P=P \mathcal{B}$ follows immediately.

We now notice that, by elementary operator theory,

$$
\forall(x, \varepsilon) \in \mathbb{R} \times \mathcal{R}, \exists K(x, \varepsilon)<1 \text { such that }\|\mathcal{B}(x) P\|_{\mathcal{L}\left(L^{2}\left(\mathcal{S}_{\varepsilon}\right)\right)} \leq K(x, \varepsilon)<1 .
$$

In the remainder, we shall assume that this constant is bounded away from 1 , as $(x, \varepsilon)$ vary. More precisely:

Hypothesis 3.3. There exists $K<1$ such that

$$
\|\mathcal{B}(x) P\|_{\mathcal{L}\left(L^{2}\left(\mathcal{S}_{\varepsilon}\right)\right)} \leq K, \quad \forall \varepsilon \in \mathcal{R}, \forall x \in \mathbb{R} .
$$


Note that Hypothesis 3.3 is equivalent to saying that

$$
|\mathcal{B}(x) u|_{L^{2}\left(\mathcal{S}_{\varepsilon}\right)} \leq K|u|_{L^{2}\left(\mathcal{S}_{\varepsilon}\right)}, \quad \forall u \in L^{2}\left(\mathcal{S}_{\varepsilon}\right) \text { such that } Q u=0 .
$$

Obviously, the hypothesis is satisfied in the case of the isotropic transmission operator $\mathcal{B}(x)=Q$. It is also satisfied if $\mathcal{B}(x)$ is bounded by a constant times $Q$ from below, as the following lemma states.

LEMma 3.4. Suppose that there exists a constant $\sigma_{0}>0$ such that

$$
\mathcal{V}(\varepsilon) \sigma\left(x, k^{\prime}, k\right) \geq \sigma_{0} \quad \forall x \in \Omega^{\alpha}, \forall k, k^{\prime} \in \mathcal{S}_{\varepsilon} \text {, a.e. } \varepsilon \in \mathcal{R} .
$$

Then, $\mathcal{B}(x)$ satisfies Hypothesis 3.3.

Proof. First, note that (3.20) and the flux conservation relation (3.5) imply that $0<$ $\sigma_{0}<1$. Let $\varphi \in L^{2}\left(\mathcal{O}^{\alpha}\right)$ be such that $\int_{\mathcal{S}_{\varepsilon}} \varphi(k)\left|v_{x}(k)\right| d N_{\varepsilon}(k)=0$, a.e. $\varepsilon \in \mathcal{R}$. We can write, for $k \in \mathcal{S}_{\varepsilon}$,

$$
\mathcal{V}(\varepsilon) \mathcal{B}(x) \varphi(k)=\int_{\mathcal{S}_{\varepsilon}}\left(\mathcal{V}(\varepsilon) \sigma\left(x, k^{\prime}, k\right)-\frac{\sigma_{0}}{2}\right) \varphi\left(k^{\prime}\right)\left|v_{x}\left(k^{\prime}\right)\right| d N_{\varepsilon}\left(k^{\prime}\right) .
$$

Since $\mathcal{V}(\varepsilon) \sigma\left(x, k^{\prime}, k\right)-\frac{\sigma_{0}}{2}>0$, and using Cauchy-Schwarz's inequality, we deduce that

$$
\begin{aligned}
\mathcal{V}(\varepsilon)^{2}|\mathcal{B}(x) \varphi(k)|^{2} \leq & \int_{\mathcal{S}_{\varepsilon}}\left(\mathcal{V}(\varepsilon) \sigma\left(x, k^{\prime}, k\right)-\frac{\sigma_{0}}{2}\right)\left|v_{x}\left(k^{\prime}\right)\right| d N_{\varepsilon}\left(k^{\prime}\right) \\
& \times \int_{\mathcal{S}_{\varepsilon}}\left(\mathcal{V}(\varepsilon) \sigma\left(x, k^{\prime}, k\right)-\frac{\sigma_{0}}{2}\right)\left|\varphi\left(k^{\prime}\right)\right|^{2}\left|v_{x}\left(k^{\prime}\right)\right| d N_{\varepsilon}\left(k^{\prime}\right) \\
\leq & \mathcal{V}(\varepsilon)\left(1-\frac{\sigma_{0}}{2}\right) \int_{\mathcal{S}_{\varepsilon}} \mathcal{V}(\varepsilon) \sigma\left(x, k^{\prime}, k\right)\left|\varphi\left(k^{\prime}\right)\right|^{2}\left|v_{x}\left(k^{\prime}\right)\right| d N_{\varepsilon}\left(k^{\prime}\right) .
\end{aligned}
$$

It follows that

$$
\int_{\mathcal{S}_{\varepsilon}}|\mathcal{B}(x) \varphi(k)|^{2}\left|v_{x}(k)\right| d N_{\varepsilon}(k) \leq\left(1-\frac{\sigma_{0}}{2}\right) \int_{\mathcal{S}_{\varepsilon}}|\varphi(k)|^{2}\left|v_{x}(k)\right| d N_{\varepsilon}(k),
$$

which completes the proof.

We close this section by giving the following technical hypothesis on the $x$ and $\varepsilon$ dependence of the operator $\mathcal{B}^{*}$.

Hypothesis 3.4. Denote by $\partial_{x} \mathcal{B}^{*}$ and $\partial_{\varepsilon} \mathcal{B}^{*}$ the operators defined by (3.14) with $\sigma$ replaced respectively by $\partial_{x} \sigma$ and $\partial_{\varepsilon} \sigma$. Then, $\partial_{x} \mathcal{B}^{*}$ and $\partial_{\varepsilon} \mathcal{B}^{*}$ are assumed to be bounded operators on $L^{2}\left(\mathcal{S}_{\varepsilon}\right)$, uniformly with respect to $(x, \varepsilon) \in \mathbb{R} \times \mathcal{R}$.

3.4. Functional setting. We define the transport operator $\mathcal{A}^{\alpha}$ by

$$
\mathcal{A}^{\alpha} u=\left(v_{x}(k) \partial_{x}+\partial_{x} V \partial_{k_{x}}\right) u
$$

on the domain

$$
H^{\alpha}(\mathcal{A}, \mathcal{B})=\left\{u \in H^{\alpha}(\mathcal{A}), \gamma_{\alpha}^{\text {out }}(u) \in L^{2}\left(\Gamma^{\alpha}\right), \gamma_{\alpha}^{\text {inc }}(u)=\mathcal{B}^{\alpha} \gamma_{\alpha}^{\text {out }}(u)\right\}
$$

where the space $H^{\alpha}(\mathcal{A})$ is given by

$$
H^{\alpha}(\mathcal{A})=\left\{u \in L^{2}\left(\mathcal{O}^{\alpha}\right), \mathcal{A}^{\alpha} u \in L^{2}\left(\mathcal{O}^{\alpha}\right)\right\}
$$

and

$$
\gamma_{\alpha}^{\text {out }}(u)=\left(\gamma_{n \alpha}^{\text {out }}(u)\right)_{n \in \mathbb{Z}}, \quad \gamma_{\alpha}^{\text {inc }}(u)=\left(\gamma_{n \alpha}^{\text {inc }}(u)\right)_{n \in \mathbb{Z}}
$$


The spaces $H^{\alpha}(\mathcal{A})$ and $H^{\alpha}(\mathcal{A}, \mathcal{B})$ are equipped with the graph norm

$$
|u|_{H^{\alpha}(\mathcal{A})}^{2}=|u|_{L^{2}\left(\mathcal{O}^{\alpha}\right)}^{2}+\left|\mathcal{A}^{\alpha} u\right|_{L^{2}\left(\mathcal{O}^{\circ}\right)}^{2} .
$$

We shall denote by $\mathcal{A}$ the bare differential operator (3.21), when no indication of the domain is needed.

We now comment on the choice of the domain of $\mathcal{A}^{\alpha}$. It is well known ([4], [32]) that the regularity $u \in H^{\alpha}(\mathcal{A})$ is not sufficient to guarantee that the traces $\gamma_{\alpha}^{\text {out }}(u)$ and $\gamma_{\alpha}^{\text {inc }}(u)$ are square integrable with respect to the measure $\left|v_{x}(k)\right| d k$. But if one of these traces is integrable, the other one is also integrable. Therefore, defining

$$
H_{0}^{\alpha}(\mathcal{A})=\left\{u \in H^{\alpha}(\mathcal{A}), \gamma_{\alpha}^{\text {out }}(u) \in L^{2}\left(\Gamma^{\alpha}\right)\right\}=\left\{u \in H^{\alpha}(\mathcal{A}), \gamma_{\alpha}^{\text {inc }}(u) \in L^{2}\left(\Gamma^{\alpha}\right)\right\}
$$

we have the following Green's formula ([4], [32]):

Lemma 3.5 (Green's formula). For $v, w$ in $H_{0}^{\alpha}(\mathcal{A})$, we have

$$
\begin{aligned}
& (\mathcal{A} u, w)_{L^{2}\left(\mathcal{O}^{\alpha}\right)}+(u, \mathcal{A} w)_{L^{2}\left(\mathcal{O}^{\alpha}\right)} \\
& =\frac{1}{\alpha}\left(\left(\gamma_{\alpha}^{\text {out }}(u), \gamma_{\alpha}^{\text {out }}(w)\right)_{L^{2}\left(\Gamma^{\alpha}\right)}-\left(\gamma_{\alpha}^{\text {inc }}(u), \gamma_{\alpha}^{\text {inc }}(w)\right)_{L^{2}\left(\Gamma^{\alpha}\right)}\right),
\end{aligned}
$$

where $(\cdot, \cdot)_{L^{2}\left(\mathcal{O}^{\alpha}\right)}$ and $(\cdot, \cdot)_{L^{2}\left(\Gamma^{\alpha}\right)}$ stand for the inner products associated with the norms of $L^{2}\left(\mathcal{O}^{\alpha}\right)$ and $L^{2}\left(\Gamma^{\alpha}\right)$.

In order to prove that $\mathcal{A}^{\alpha}$ with domain $H^{\alpha}(\mathcal{A}, \mathcal{B})$ generates a strongly continuous semigroup (which is enough to prove existence of solutions of (2.6)-(2.7)), the closedness of $H^{\alpha}(\mathcal{A}, \mathcal{B})$ with respect to the graph norm of $\mathcal{A}^{\alpha}$ is needed. A way of proving such a property is to control $\left|\gamma_{\alpha}^{\text {out }}(u)\right|_{L^{2}\left(\Gamma^{\alpha}\right)}$ in terms of the graph norm. By applying the Green's formula (3.23) with $w=u$, it is easy to get the estimate

$$
(1-\|\mathcal{B}\|)\left|\gamma_{\alpha}^{\text {out }}(u)\right|_{L^{2}\left(\Gamma^{\alpha}\right)}^{2} \leq \alpha|u|_{H^{\alpha}(\mathcal{A})}^{2} .
$$

Unfortunately, since $\mathcal{B}$ leaves invariant the set of constant functions on the energy shells $\mathcal{S}_{\varepsilon}$, the norm of $\mathcal{B}$ is equal to one, and the above inequality does not provide the sought control of $\left|\gamma_{\alpha}^{\text {out }}(u)\right|_{L^{2}\left(\Gamma^{\alpha}\right)}$. In order to deal with this problem, we introduce the operator

$$
\mathcal{B}_{\eta}=P \mathcal{B}+\frac{1}{1+\eta} Q, \quad \eta>0
$$

and the operator $\mathcal{A}_{\eta}^{\alpha}=\mathcal{A}$ with domain $H^{\alpha}\left(\mathcal{A}, \mathcal{B}_{\eta}\right)$, defined by

$$
H^{\alpha}\left(\mathcal{A}, \mathcal{B}_{\eta}\right)=\left\{u \in H^{\alpha}(\mathcal{A}), \gamma_{\alpha}^{\text {out }}(u) \in L^{2}\left(\Gamma^{\alpha}\right), \gamma_{\alpha}^{\text {inc }}(u)=\mathcal{B}_{\eta} \gamma_{\alpha}^{\text {out }}(u)\right\}
$$

Straightforward computations using Lemma 3.3 lead to the following identity satisfied by any function $\phi \in L^{2}\left(\Gamma^{\alpha}\right)$ :

$$
\left\|\mathcal{B}_{\eta}(\phi)\right\|^{2}=\left(1-\frac{1}{(1+\eta)^{2}}\right)\|P \mathcal{B}(\phi)\|^{2}+\frac{1}{(1+\eta)^{2}}\|\mathcal{B}(\phi)\|^{2} .
$$

This implies, for $\eta$ small enough,

$$
\left\|\mathcal{B}_{\eta}\right\|_{\mathcal{L}\left(L^{2}(B)\right)} \leq 1-C \eta<1, \quad \forall 0<\eta<\eta_{0},
$$

and the above argument leads to the closedness of the operator $\mathcal{A}_{\eta}^{\alpha}$. In the next section, we prove that it generates a strongly continuous semigroup of contractions. 
3.5. $\mathcal{A}_{\eta}^{\alpha}$ generates a continuous semigroup of contractions. In this section we are concerned with the properties of the operator $\mathcal{A}_{\eta}^{\alpha}$. Throughout this section and unless otherwise stated, $\eta$ is a fixed positive real number.

Proposition 3.6. $\mathcal{A}_{\eta}^{\alpha}$, with domain $H^{\alpha}\left(\mathcal{A}, \mathcal{B}_{\eta}\right)$, is a maximal accretive operator.

Proof. That $\mathcal{A}_{\eta}^{\alpha}$ is accretive follows immediately from Green's formula and (3.26), which lead to

$$
2 \alpha\left(\mathcal{A}_{\eta}^{\alpha} u, u\right)_{L^{2}\left(\mathcal{O}^{\alpha}\right)} \geq\left(1-\left|\mathcal{B}_{\eta}\right|_{\mathcal{L}\left(L^{2}(B)\right)}^{2}\right)\left|\gamma_{\alpha}^{\text {out }}(u)\right|_{L^{2}\left(\Gamma^{\alpha}\right)}^{2} \geq 0
$$

We now prove that $\mathcal{A}_{\eta}^{\alpha}$ is maximal, i.e., that for all $f$ in $L^{2}\left(\mathcal{O}^{\alpha}\right)$, there exists $u \in$ $H^{\alpha}\left(\mathcal{A}, \mathcal{B}_{\eta}\right)$ such that

$$
u+\mathcal{A} u=f .
$$

Let $f$ be a given function in $L^{2}\left(\mathcal{O}^{\alpha}\right)$. We search for $u$ by means of a fixed point method. We define the application $T_{\eta}$ on $L^{2}\left(\Gamma^{\alpha}\right)$ as follows: for $\varphi \in L^{2}\left(\Gamma^{\alpha}\right)$, let $v$ be the solution in $H^{\alpha}(\mathcal{A})$ of

$$
\left\{\begin{array}{l}
v+\mathcal{A} v=f \\
\gamma_{\alpha}^{\text {inc }}(v)=\varphi
\end{array}\right.
$$

(such a solution exists and is unique in $H^{\alpha}(\mathcal{A})$, see [4] or [32]). Then $\gamma_{\alpha}^{\text {out }}(v)$ lies in $L^{2}\left(\Gamma^{\alpha}\right)$, and we set

$$
T_{\eta}(\varphi)=\mathcal{B}_{\eta} \gamma_{\alpha}^{\text {out }}(v)
$$

It is readily seen that a solution of (3.27) corresponds to a fixed point for the application $T_{\eta}$, so that Proposition 3.6 is a consequence of

Lemma 3.7. $T_{\eta}$ is a contraction on $L^{2}\left(\Gamma^{\alpha}\right)$, with Lipschitz constant $\left\|\mathcal{B}_{\eta}\right\|_{\mathcal{L}\left(L^{2}(B)\right)}$; hence it admits a unique fixed point.

Proof. Let $\varphi_{1}$ and $\varphi_{2}$ be in $L^{2}\left(\Gamma^{\alpha}\right)$, and $v_{1}, v_{2}$ be the associated solutions of (3.28). Then, we have

$$
v_{2}-v_{1}+\mathcal{A}\left(v_{2}-v_{1}\right)=0 \quad \text { and } \quad \gamma_{\alpha}^{\text {inc }}\left(v_{2}-v_{1}\right)=\varphi_{2}-\varphi_{1} .
$$

Multiplying by $v_{2}-v_{1}$ in the previous equation, and integrating, we get

$$
2\left|v_{2}-v_{1}\right|_{L^{2}\left(\mathcal{O}^{\alpha}\right)}^{2}+\left|\gamma_{\alpha}^{\text {out }}\left(v_{2}-v_{1}\right)\right|_{L^{2}\left(\Gamma^{\alpha}\right)}^{2}=\left|\gamma_{\alpha}^{\text {inc }}\left(v_{2}-v_{1}\right)\right|_{L^{2}\left(\Gamma^{\alpha}\right)}^{2},
$$

which implies

$$
\begin{aligned}
\left|T_{\eta}\left(\varphi_{2}\right)-T_{\eta}\left(\varphi_{1}\right)\right|_{L^{2}\left(\Gamma^{\alpha}\right)} & \leq\left\|\mathcal{B}_{\eta}\right\|_{\mathcal{L}\left(L^{2}(B)\right)}\left|\gamma_{\alpha}^{\text {out }}\left(v_{2}-v_{1}\right)\right|_{L^{2}\left(\Gamma^{\alpha}\right)} \\
& \leq\left\|\mathcal{B}_{\eta}\right\|_{\mathcal{L}\left(L^{2}(B)\right)}\left|\varphi_{2}-\varphi_{1}\right|_{L^{2}\left(\Gamma^{\alpha}\right)}
\end{aligned}
$$

By applying Hille-Yosida's theorem (see [10]), we obtain

Lemma 3.8. For all $\eta>0$, for all $F_{\eta} \in H^{\alpha}\left(\mathcal{A}, \mathcal{B}_{\eta}\right)$, there exists a unique function $f_{\eta}^{\alpha} \in \mathcal{C}\left([0, T] ; H^{\alpha}\left(\mathcal{A}, \mathcal{B}_{\eta}\right)\right) \cap \mathcal{C}^{1}\left([0, T] ; L^{2}\left(\mathcal{O}^{\alpha}\right)\right)$, solving

$$
\begin{aligned}
\alpha \partial_{t} f_{\eta}^{\alpha}+\mathcal{A} f_{\eta}^{\alpha} & =0, \\
\left.f_{\eta}^{\alpha}\right|_{t=0} & =F_{\eta} .
\end{aligned}
$$


Moreover, we have the following estimates:

$$
\left|f_{\eta}^{\alpha}\right|_{L^{2}\left(\mathcal{O}^{\alpha}\right)} \leq\left|F_{\eta}\right|_{L^{2}\left(\mathcal{O}^{\alpha}\right)}, \quad\left|\alpha \partial_{t} f_{\eta}^{\alpha}\right|_{L^{2}\left(\mathcal{O}^{\alpha}\right)}=\left|\mathcal{A} f_{\eta}^{\alpha}\right|_{L^{2}\left(\mathcal{O}^{\alpha}\right)} \leq\left|\mathcal{A} F_{\eta}\right|_{L^{2}\left(\mathcal{O}^{\alpha}\right)} .
$$

Since we are aiming at approximating the solution of problem (2.6)-(2.9) by a sequence of solutions $f_{\eta}$ given by the previous lemma, we need the following result.

Lemma 3.9. Let $F_{I}$ be as in Hypothesis 2.2. There exists a sequence $(\eta)$ tending to zero and $\left(F_{\eta}\right)_{\eta>0}$ such that $F_{\eta} \in H^{\alpha}\left(\mathcal{A}, \mathcal{B}_{\eta}\right)$ and

$$
F_{n} \rightarrow F_{I}, \quad \mathcal{A} F_{\eta} \rightarrow \mathcal{A} F_{I} \quad \text { weakly in } L^{2}\left(\mathcal{O}^{\alpha}\right) .
$$

Proof. We define the function $F_{\eta}$ as the solution of

$$
F_{\eta}+\mathcal{A} F_{\eta}=F_{I}+\mathcal{A} F_{I}, \quad F_{\eta} \in H^{\alpha}\left(\mathcal{A}, \mathcal{B}_{\eta}\right) .
$$

Such a function exists for all $\eta>0$ since $\mathcal{A}_{\eta}^{\alpha}$ is maximal; moreover, we have

$$
\begin{aligned}
\left|F_{\eta}\right|_{L^{2}\left(\mathcal{O}^{\alpha}\right)} & \leq\left|F_{I}+\mathcal{A} F_{I}\right|_{L^{2}\left(\mathcal{O}^{\alpha}\right)} \\
\left|\mathcal{A} F_{\eta}\right|_{L^{2}\left(\mathcal{O}^{\alpha}\right)} & \leq\left|F_{\eta}\right|+\left|F_{I}+\mathcal{A} F_{I}\right|_{L^{2}\left(\mathcal{O}^{\alpha}\right)} \leq 2\left|F_{I}+\mathcal{A} F_{I}\right|_{L^{2}\left(\mathcal{O}^{\alpha}\right)} .
\end{aligned}
$$

The first estimate follows from the monotonicity of $\mathcal{A}_{\eta}^{\alpha}$, and the second one is obvious. As a consequence, there exists $F \in H^{\alpha}(\mathcal{A})$ such that $F_{\eta} \rightarrow F$, and $\mathcal{A} F_{\eta} \rightarrow \mathcal{A} F$ in $L^{2}\left(\mathcal{O}^{\alpha}\right)$ weak. It remains to prove that $F=F_{I}$. If we prove that $F \in H^{\alpha}(\mathcal{A}, \mathcal{B})$, the result follows, since $(\mathcal{A} u, u)_{L^{2}\left(\mathcal{O}^{\alpha}\right)} \geq 0$ for $u \in H^{\alpha}(\mathcal{A}, \mathcal{B})$, and $F-F_{I} \in H^{\alpha}(\mathcal{A}, \mathcal{B})$. Thus, it is enough to prove that $\gamma_{\alpha}^{\text {out }}(F)$ lies in $L^{2}\left(\Gamma^{\alpha}\right)$. To this aim, let us define $\varphi_{0}=\gamma_{\alpha}^{\text {inc }}\left(F_{I}\right)$. Then, it is readily seen that

$$
T_{\eta}\left(\varphi_{0}\right)=\frac{1}{1+\eta} \varphi_{0}
$$

where $T_{\eta}$ is a contraction introduced in the proof of Proposition 3.6. Since $\gamma_{\alpha}^{\text {inc }}\left(F_{\eta}\right)$ is the unique fixed point of $T_{\eta}$, we have the following identity:

$$
\gamma_{\alpha}^{\text {inc }}\left(F_{\eta}\right)-\varphi_{0}=T_{\eta}\left(\gamma_{\alpha}^{\text {inc }}\left(F_{\eta}\right)\right)-T_{\eta}\left(\varphi_{0}\right)-\frac{\eta}{1+\eta} \varphi_{0}
$$

which immediately leads to

$$
\left|\gamma_{\alpha}^{\text {inc }}\left(F_{\eta}\right)-\varphi_{0}\right|_{L^{2}\left(\Gamma^{\alpha}\right)} \leq \frac{\eta}{1+\eta} \frac{\left|\varphi_{0}\right|_{L^{2}\left(\Gamma^{\alpha}\right)}}{1-\left\|T_{\eta}\right\|} \leq \frac{\eta}{1+\eta} \frac{\left|\varphi_{0}\right|_{L^{2}\left(\Gamma^{\circ}\right)}}{C \eta} \leq C,
$$

which proves the boundedness of $\gamma_{\alpha}^{\text {inc }}\left(F_{\eta}\right)$ in $L^{2}\left(\Gamma^{\alpha}\right)$.

Estimates (3.30) allow us to take the limit $\eta \rightarrow 0$ in Eq. (3.29). In order to take the limit in the equality $\gamma_{\alpha}^{\text {inc }}\left(f_{\eta}^{\alpha}\right)=\mathcal{B}_{\eta} \gamma_{\alpha}^{\text {out }}\left(f_{\eta}^{\alpha}\right)$, we need some trace estimates. This is the aim of the next section.

3.6. $L^{2}$ trace estimates. In this section, we establish the control of $\gamma_{\alpha}^{\text {out }}(u), \gamma_{\alpha}^{\text {inc }}(u)$ in terms of $|u|_{H^{\alpha}(\mathcal{A})}$. Let us now denote by $P$ and $Q$ the orthogonal projections of $L^{2}\left(\Gamma^{\alpha}\right)$ defined for $u=\left(u_{\alpha n}\right)_{n \in \mathbb{Z}}$ by

$$
Q u=\left(Q u_{\alpha n}\right)_{n \in \mathbb{Z}}, \quad P u=\left(P u_{\alpha n}\right)_{n \in \mathbb{Z}} .
$$

Then we have the following proposition. 
Proposition 3.10. For all $u \in H^{\alpha}\left(\mathcal{A}, \mathcal{B}_{\eta}\right)$, we have

$$
\left|P \gamma_{\alpha}^{\text {inc }}(u)\right|_{L^{2}\left(\Gamma^{\alpha}\right)}^{2} \leq\left|P \gamma_{\alpha}^{\text {out }}(u)\right|_{L^{2}\left(\Gamma^{\alpha}\right)}^{2} \leq \frac{2 \alpha}{1-K^{2}}(\mathcal{A} u, u)_{L^{2}\left(\mathcal{O}^{\alpha}\right)}
$$

(ii) $\quad(1+\eta) Q \gamma_{\alpha}^{\mathrm{inc}}(u)=Q \gamma_{\alpha}^{\mathrm{out}}(u):=q^{\alpha}(u)=\left(q_{n \alpha}\right)_{n \in \mathbb{Z}}$

and we have

$$
\left|q^{\alpha}(u)\right|_{L_{\delta}^{2}\left(\Gamma^{\alpha}\right)}^{2} \leq C_{1} \alpha|u|_{H^{\alpha}(A)}^{2}+C_{2}\left(1+\frac{\alpha}{\delta}\right)|u|_{L^{2}\left(\mathcal{O}^{\alpha}\right)}^{2}
$$

Proof of Proposition 3.10. (i) Let $u \in H^{\alpha}\left(\mathcal{A}, \mathcal{B}_{\eta}\right)$. Then by Green's formula (3.23), we have

$$
\begin{aligned}
2 \alpha(\mathcal{A} u, u)_{L^{2}\left(\mathcal{O}^{\alpha}\right)}= & \left|\gamma_{\alpha}^{\text {out }}(u)\right|_{L^{2}\left(\Gamma^{\alpha}\right)}^{2}-\left|\gamma_{\alpha}^{\text {inc }}(u)\right|_{L^{2}\left(\Gamma^{\alpha}\right)}^{2} \\
= & \left|\gamma_{\alpha}^{\text {out }}(u)\right|_{L^{2}\left(\Gamma^{\alpha}\right)}^{2}-\left|\mathcal{B}_{\eta} \gamma_{\alpha}^{\text {out }}(u)\right|_{L^{2}\left(\Gamma^{\alpha}\right)}^{2} \\
= & \left|P \gamma_{\alpha}^{\text {out }}(u)\right|_{L^{2}\left(\Gamma^{\alpha}\right)}^{2}-\left|\mathcal{B}_{\eta} P \gamma_{\alpha}^{\text {out }}(u)\right|_{L^{2}\left(\Gamma^{\alpha}\right)}^{2} \\
& \quad+\left(1-\frac{1}{(1+\eta)^{2}}\right)\left|Q \gamma_{\alpha}^{\text {out }}(u)\right|_{L^{2}\left(\Gamma^{\alpha}\right)}^{2} \\
\geq & \left(1-K^{2}\right)\left|P \gamma_{\alpha}^{\text {out }}(u)\right|_{L^{2}\left(\Gamma^{\alpha}\right)}^{2},
\end{aligned}
$$

which shows the second inequality of (3.32), the first one obviously following from (3.11).

(ii) Define $\phi^{\alpha}(x)$ by

$$
\phi^{\alpha}(x)=\frac{2}{\alpha}\left(x-\left(n-\frac{1}{2}\right) \alpha\right), \quad x \in((n-1) \alpha, n \alpha) .
$$

Note that $\left|\phi^{\alpha}\right|_{L^{\infty}\left(\Omega^{\alpha}\right)}=1$, that $\gamma_{n \alpha}^{-}\left(\phi^{\alpha}\right)=1, \gamma_{(n-1) \alpha}^{+}\left(\phi^{\alpha}\right)=-1$ and that $\frac{\partial \phi^{\alpha}}{\partial x}=\frac{2}{\alpha}$. The control of $Q \gamma_{\alpha}^{\text {out }}(u)=Q \gamma_{\alpha}^{\text {inc }}(u)$ is obtained by multiplying $\mathcal{A} u$ by $\operatorname{sgn}\left(v_{x}\right) u$ and integrating with respect to $x$ and $k$. This method obviously breaks down for small $v_{x}(k)$ if the electric field $\partial_{x} V$ is nonzero, because $\operatorname{sgn}\left(v_{x}\right)$ is not differentiable. However, by regularizing the sign function and using (3.4), it is possible to limit the breakdown of the method to the boundary of the energy range $\mathcal{R}$. This is why we only have estimates of the projections $Q \gamma_{\alpha}^{\text {out }}(u)=Q \gamma_{\alpha}^{\text {inc }}(u)$ in the weighted spaces $L_{\delta}^{2}\left(\Gamma^{\alpha}\right), \forall \delta>0$. Using 
Green's formula as before, we get for all $\delta>0$ :

$$
\begin{aligned}
2(\mathcal{A} & \left.u, u s^{\delta}\left(v_{x}(k)\right) \phi^{\alpha}\right)_{L^{2}\left(\mathcal{O}^{\alpha}\right)}+\frac{2}{\alpha} \int_{\mathcal{O}^{\alpha}}|u|^{2} w^{\delta}(k) d x d k \\
& +\int_{\mathcal{O}^{\alpha}} \partial_{x} V|u|^{2} \partial_{k_{x}}\left(s^{\delta}\left(v_{x}(k)\right)\right) \phi^{\alpha} d x d k \\
= & \sum_{n \in \mathbb{Z}} \int_{B}\left(\left|\gamma_{n \alpha}^{-}(u)\right|^{2}+\left|\gamma_{(n-1) \alpha}^{+}(u)\right|^{2}\right) w^{\delta}(k) d k \\
= & \sum_{n \in \mathbb{Z}} \int_{B}\left(\left|\gamma_{n \alpha}^{\text {out }}(u)\right|^{2}+\left|\gamma_{n \alpha}^{\text {inc }}(u)\right|^{2}\right) w^{\delta}(k) d k \\
= & \left(1+\frac{1}{(1+\eta)^{2}}\right) \sum_{n \in \mathbb{Z}} \int_{B}\left|q_{n \alpha}(u)\right|^{2} w^{\delta}(k) d k \\
& +\sum_{n \in \mathbb{Z}} \int_{B}\left(\left|P \gamma_{n \alpha}^{\text {out }}(u)\right|^{2}+\left|P \gamma_{n \alpha}^{\text {inc }}(u)\right|^{2}\right) w^{\delta}(k) d k \\
& +2 \sum_{n \in \mathbb{Z}} \int_{B}\left(P \gamma_{n \alpha}^{\text {out }}(u)+\frac{1}{1+\eta} P \gamma_{n \alpha}^{\text {inc }}(u)\right) q_{n \alpha}(u) w^{\delta}(k) d k .
\end{aligned}
$$

Moreover, the last term in (3.36) can be bounded by

$$
\frac{4}{\alpha}\left(\left|P \gamma_{\alpha}^{\text {inc }}(u)\right|_{L^{2}\left(\Gamma^{\alpha}\right)}^{2}+\left|P \gamma_{\alpha}^{\text {out }}(u)\right|_{L^{2}\left(\Gamma^{\alpha}\right)}^{2}\right)+\frac{1}{4} \sum_{n \in \mathbb{Z}} \int_{B}\left|q_{n \alpha}(u)\right|^{2} w^{\delta}(k) d k .
$$

Therefore, we get

$$
\begin{aligned}
& \left(\frac{3}{4}+\frac{1}{(1+\eta)^{2}}\right) \sum_{n \in \mathbb{Z}} \int_{B}\left|q_{n \alpha}(u)\right|^{2} w^{\delta}(k) d k \leq 2\left(\mathcal{A}^{\alpha} u, u s^{\delta}\left(v_{x}(k)\right) \phi^{\alpha}\right)_{L^{2}\left(\mathcal{O}^{\alpha}\right)}+\frac{2}{\alpha}|u|_{L^{2}\left(\mathcal{O}^{\alpha}\right)}^{2} \\
& \quad+\left|\partial_{x} V\right|_{L^{\infty}}\left|\partial_{k_{x}}\left(s^{\delta}\left(v_{x}(k)\right)\right)\right|_{L^{\infty}}|u|_{L^{2}\left(\mathcal{O}^{\alpha}\right)}^{2}+\frac{3}{\alpha}\left(\left|P \gamma_{\alpha}^{\text {inc }}(u)\right|_{L^{2}\left(\Gamma^{\alpha}\right)}^{2}+\left|P \gamma_{\alpha}^{\text {out }}(u)\right|_{L^{2}\left(\Gamma^{\alpha}\right)}^{2}\right) .
\end{aligned}
$$

Estimate (3.34) follows immediately from (3.37) after multiplying by $\alpha$ and using the boundedness of $\phi^{\alpha}$ and $v_{x}(k)$ (since $v_{x}(k)$ is continuous and defined on the compact set $B)$ and the following inequality:

$$
\left|\partial_{k_{x}}\left(s^{\delta}\left(v_{x}(k)\right)\right)\right|_{L^{\infty}} \leq \frac{C}{\delta}\left|\varepsilon^{\prime \prime}\right|_{L^{\infty}}
$$

3.7. Existence of a solution to the problem (2.6)-(2.7). We can now prove the first part of Theorem 2.1. More precisely, we prove:

Proposition 3.11. Under Hypotheses $2.1,2.2,3.1,3.2,3.3$, there exists a solution $f^{\alpha}$ to problem (2.6), (2.7), such that $f^{\alpha} \in L^{\infty}\left(0, T ; L^{2}\left(\mathcal{O}^{\alpha}\right)\right), \mathcal{A} f^{\alpha} \in L^{\infty}\left(0, T ; L^{2}\left(\mathcal{O}^{\alpha}\right)\right)$, $P \gamma_{\alpha}^{\text {out }}\left(f^{\alpha}\right) \in L^{\infty}\left(0, T ; L^{2}\left(\Gamma^{\alpha}\right)\right), Q \gamma_{\alpha}^{\text {out }}\left(f^{\alpha}\right) \in L^{\infty}\left(0, T ; L_{\delta}^{2}\left(\Gamma^{\alpha}\right)\right)$, for all $\delta>0$, and the boundary condition is satisfied in the sense that

$$
P \gamma_{\alpha}^{\text {inc }}\left(f^{\alpha}\right)=\mathcal{B P} \gamma_{\alpha}^{\text {out }}\left(f^{\alpha}\right), \quad Q \gamma_{\alpha}^{\text {inc }}\left(f^{\alpha}\right)=Q \gamma_{\alpha}^{\text {out }}\left(f^{\alpha}\right) .
$$


Proof. Let $f_{\eta}^{\alpha}$ be the solution to the approximate problem

$$
\left\{\begin{array}{l}
\alpha \partial_{t} f_{\eta}^{\alpha}+\mathcal{A} f_{\eta}^{\alpha}=0, \\
f_{\eta}^{\alpha} \in H^{\alpha}\left(\mathcal{A}, \mathcal{B}_{\eta}\right), \\
\left.f_{\eta}^{\alpha}\right|_{t=0}=F_{\eta},
\end{array}\right.
$$

where $F_{\eta}$ is the function constructed in Lemma 3.9. Then, thanks to the estimates (3.30), there exists $f^{\alpha} \in H^{\alpha}(\mathcal{A})$ such that, up to a subsequence, $f_{\eta}^{\alpha}$ converges towards $f^{\alpha}$ in $L^{\infty}\left([0, T], L^{2}\left(\mathcal{O}^{\alpha}\right)\right)$ weak star, and $\mathcal{A} f_{\eta}^{\alpha}$ converges towards $\mathcal{A} f^{\alpha}$ in $L^{\infty}\left([0, T], L^{2}\left(\mathcal{O}^{\alpha}\right)\right)$ weak star. Then $\partial_{t} f^{\alpha} \in L^{2}\left(\mathcal{O}^{\alpha}\right)$, and

$$
\alpha \partial_{t} f^{\alpha}+\mathcal{A} f^{\alpha}=0 .
$$

Moreover, Proposition 3.10, Lemma 3.8 and Lemma 3.9 give

$$
\begin{aligned}
& \left|P \gamma_{\alpha}^{\text {inc }}\left(f_{\eta}^{\alpha}\right)\right|_{L^{2}\left(\Gamma^{\alpha}\right)}^{2} \leq\left|P \gamma_{\alpha}^{\text {out }}\left(f_{\eta}^{\alpha}\right)\right|_{L^{2}\left(\Gamma^{\alpha}\right)}^{2} \leq \frac{2 \alpha}{1-K^{2}}\left|F_{I}\right|_{H^{\alpha}(\mathcal{A})}^{2}, \\
& (1+\eta)^{2}\left|Q \gamma_{\alpha}^{\text {inc }}\left(f_{\eta}^{\alpha}\right)\right|_{L_{\delta}^{2}\left(\Gamma^{\alpha}\right)}^{2}=\left|Q \gamma_{\alpha}^{\text {out }}\left(f_{\eta}^{\alpha}\right)\right|_{L_{\delta}^{2}\left(\Gamma^{\alpha}\right)}^{2} \\
& \leq C_{1} \alpha\left|F_{I}\right|_{H^{\alpha}(A)}^{2}+C_{2}\left(1+\frac{\alpha}{\delta}\right)\left|F_{I}\right|_{L^{2}\left(\mathcal{O}^{\alpha}\right)}^{2} .
\end{aligned}
$$

Then, we can assume that $P \gamma_{\alpha}^{\text {out }}\left(f_{\eta}^{\alpha}\right), P \gamma_{\alpha}^{\text {inc }}\left(f_{\eta}^{\alpha}\right)$, and $Q \gamma_{\alpha}^{\text {out }}\left(f_{\eta}^{\alpha}\right), Q \gamma_{\alpha}^{\text {inc }}\left(f_{\eta}^{\alpha}\right)$ weakly converge respectively in $L^{\infty}\left(0, T ; L^{2}\left(\Gamma^{\alpha}\right)\right)$ and $L^{\infty}\left(0, T ; L^{2}\left(\Gamma^{\alpha}\right)\right)$. By a classical result (see [4], [32]), their limits are $P \gamma_{\alpha}^{\text {out }}\left(f^{\alpha}\right), P \gamma_{\alpha}^{\text {inc }}\left(f^{\alpha}\right)$, and $Q \gamma_{\alpha}^{\text {out }}\left(f^{\alpha}\right), Q \gamma_{\alpha}^{\text {inc }}\left(f^{\alpha}\right)$ in the distributional sense and we deduce that the traces of $f^{\alpha}$ satisfy

$$
\begin{aligned}
& P \gamma_{\alpha}^{\text {inc }}\left(f^{\alpha}\right), P \gamma_{\alpha}^{\text {out }}\left(f^{\alpha}\right) \in L^{\infty}\left(0, T ; L^{2}\left(\Gamma^{\alpha}\right)\right), \\
& Q \gamma_{\alpha}^{\text {inc }}\left(f^{\alpha}\right), Q \gamma_{\alpha}^{\text {out }}\left(f^{\alpha}\right) \in L^{\infty}\left(0, T ; L_{\delta}^{2}\left(\Gamma^{\alpha}\right)\right), \quad \forall \delta>0,
\end{aligned}
$$

and

$$
P \gamma_{\alpha}^{\text {inc }}\left(f^{\alpha}\right)=\mathcal{B P} \gamma_{\alpha}^{\text {out }}\left(f^{\alpha}\right), \quad Q \gamma_{\alpha}^{\text {inc }}\left(f^{\alpha}\right)=Q \gamma_{\alpha}^{\text {out }}\left(f^{\alpha}\right),
$$

which concludes the proof.

REMARK 3.12. Since $Q \gamma_{\alpha}^{\text {out }}(u)$ only depends on the energy, the condition $Q \gamma_{\alpha}^{\text {out }}(u) \in$ $L_{\delta}^{2}\left(\Gamma^{\alpha}\right)$ means that

$$
\sum_{n \in \mathbb{Z}} \alpha \int_{\mathcal{R}}\left|Q \gamma_{n \alpha}^{\text {out }}(u)(\varepsilon)\right|^{2} \int_{\mathcal{S}_{\varepsilon}} w^{\delta}(k) d N_{\varepsilon}(k) d \varepsilon<\infty .
$$

According to Lemma 3.1 , for any compact subset $K$ of $\stackrel{\circ}{\mathcal{R}}$, there exists a constant $C_{K, \delta}$ such that

$$
\left|Q \gamma_{n \alpha}^{\text {out }}(u)\right|_{L^{2}(K)} \leq C_{K, \delta}\left|Q \gamma_{n \alpha}^{\text {out }}(u)\right|_{L_{\delta}^{2}(K)} .
$$

In particular, this implies that $Q \gamma_{n \alpha}^{\text {out }}(u)$ lies in $L^{2}\left(\mathcal{S}_{\varepsilon}\right)$ for almost every (later abbreviated by a.e.) $\varepsilon \in \stackrel{\circ}{\mathcal{R}}$.

Moreover, we have the following estimates, which will be useful later on:

Lemma 3.13. There exists a constant $C$ independent of $\alpha$ such that

$$
\int_{0}^{T}\left|P \gamma_{\alpha}^{\text {out }}\left(f^{\alpha}(t)\right)\right|_{L^{2}\left(\Gamma^{\alpha}\right)}^{2} d t \leq C \alpha^{2}\left|F_{I}\right|_{L^{2}(\mathbb{R} \times \mathcal{R})}^{2} .
$$


Proof. Multiplying by $f_{\eta}^{\alpha}$ the equality $\alpha \partial_{t} f_{\eta}^{\alpha}+\mathcal{A}^{\alpha} f_{\eta}^{\alpha}=0$ and integrating, we get

$$
\alpha\left|f_{\eta}^{\alpha}(t)\right|_{L^{2}\left(\mathcal{O}^{\alpha}\right)}^{2}+2 \int_{0}^{t}\left(\mathcal{A}^{\alpha} f_{\eta}^{\alpha}(s), f_{\eta}^{\alpha}(s)\right)_{L^{2}\left(\mathcal{O}^{\alpha}\right)} d s \leq \alpha\left|F_{\eta}\right|_{L^{2}\left(\mathcal{O}^{\alpha}\right)}^{2} .
$$

Then (3.35) and passing to the limit $\eta \rightarrow 0$ give the result.

4. Convergence towards the macroscopic model. In this section, we prove the convergence part of Theorem 2.1, according to the scheme outlined in the introduction.

4.1. $L^{2}$ estimates. Let us summarize the $L^{2}$ estimates deduced from the previous section.

LEMma 4.1. The solution $f^{\alpha}$ of problem (2.6)-(2.7) constructed in the previous section 292 satisfies:

$$
\begin{aligned}
\left|f^{\alpha}\right|_{C^{0}\left([0, T] ; L^{2}\left(\mathcal{O}^{\alpha}\right)\right)} & \leq\left|F_{I}\right|_{L^{2}(\mathbb{R} \times \mathcal{R})}, \\
\left|\mathcal{A}^{\alpha} f^{\alpha}\right|_{C^{0}\left([0, T] ; L^{2}\left(\mathcal{O}^{\alpha}\right)\right)} & \leq\left|\mathcal{A}^{\alpha} F_{I}\right|_{L^{2}(\mathbb{R} \times \mathcal{R})}, \\
\left|P \gamma_{\alpha}^{\text {out }}\left(f^{\alpha}(t)\right)\right|_{L^{2}\left(\Gamma^{\alpha}\right)}^{2} & \leq C \alpha\left|F_{I}\right|_{H^{\alpha}(\mathcal{A})}^{2}, \\
\int_{0}^{T}\left|P \gamma_{\alpha}^{\text {out }}\left(f^{\alpha}(t)\right)\right|_{L^{2}\left(\Gamma^{\alpha}\right)}^{2} d t & \leq C \alpha^{2}\left|F_{I}\right|_{L^{2}(\mathbb{R} \times \mathcal{R})}^{2}, \\
\int_{0}^{T}\left|P \gamma_{\alpha}^{\text {inc }}\left(f^{\alpha}(t)\right)\right|_{L^{2}\left(\Gamma^{\alpha}\right)}^{2} d t & \leq C \alpha^{2}\left|F_{I}\right|_{L^{2}(\mathbb{R} \times \mathcal{R})}^{2}, \\
\int_{0}^{T}\left|q^{\alpha}\left(f^{\alpha}(t)\right)\right|_{L_{\delta}^{2}\left(\Gamma^{\alpha}\right)}^{2} d t & \leq C_{\delta}\left|F_{I}\right|_{H}^{\alpha}(\mathcal{A}),
\end{aligned}
$$

where from now on, $C$ denotes generic constants independent of $\alpha$ and of the data.

We immediately deduce from the lemma that there exists a subsequence (still denoted by $\left.f^{\alpha}\right)$ and a function $f^{0}$ in $L^{\infty}\left(0, T ; L^{2}(\mathbb{R} \times B)\right)$ such that

$$
f^{\alpha} \rightarrow f^{0} \text { in } L^{\infty}\left(0, T, L^{2}(\mathbb{R} \times B)\right) \text { weak star }
$$

when $\alpha \rightarrow 0$.

In view of Lemma 4.1 , it is reasonable to expect that the traces $\gamma_{\alpha}^{\text {inc }}\left(f^{\alpha}\right)$ and $\gamma_{\alpha}^{\text {out }}\left(f^{\alpha}\right)$ converge to functions of the energy only. In the next section, we prove that this property remains true for $f^{\alpha}$.

4.2. $f^{0}$ is a function of the energy. Define $\tilde{f}^{\alpha}(x, k, t)$ as the piecewise constant function (with respect to $x$ ) such that

$$
\tilde{f}^{\alpha}(x, k, t)= \begin{cases}\gamma_{n \alpha}^{-}\left(f^{\alpha}\right)(k, t), & x \in\left[\left(n-\frac{1}{2}\right) \alpha, n \alpha\right], \\ \gamma_{n \alpha}^{+}\left(f^{\alpha}\right)(k, t), & x \in\left[n \alpha,\left(n+\frac{1}{2}\right) \alpha\right] .\end{cases}
$$

From (4.4), (4.5), and (4.6), it is readily seen that $\tilde{f}^{\alpha}$ is bounded in $L^{2}(] 0, T\left[\times \mathbb{R}, L_{\delta}^{2}(B)\right)$, $\forall \delta>0$. Therefore, up to a diagonal extraction of a subsequence, there exists a function $\tilde{f}^{0}$ in $L^{2}(] 0, T\left[\times \mathbb{R}, L_{\delta}^{2}(B)\right)$ such that

$$
\tilde{f}^{\alpha} \rightarrow \tilde{f}^{0} \text { in } L^{2}(] 0, T\left[\times \mathbb{R}, L_{\delta}^{2}(B)\right) \text { weak, } \quad \forall \delta>0 .
$$


Furthermore, estimates (4.4) and (4.5) show that there exists a function $F(x, \varepsilon, t)$ such that

$$
\tilde{f}^{0}(x, k, t)=F(x, \varepsilon(k), t) .
$$

Lemma 4.2. Let $f^{0}$ be the limit of the sequence $f^{\alpha}$. Then

$$
f^{0}(x, k, t)=F(x, \varepsilon(k), t) .
$$

Proof. It is sufficient to prove that $f^{\alpha}-\tilde{f}^{\alpha} \rightarrow 0$ as $\alpha \rightarrow 0$ in the distributional sense. Let $\phi$ be a test function in $\mathcal{D}(] 0, T[\times \mathbb{R} \times B)$, such that the set $\{\varepsilon(k),(x, k, t) \in \operatorname{supp}(\phi)\}$ is a compact set of $\stackrel{\circ}{\mathcal{R}}$. Defining

$$
\psi^{\alpha}(x)=\left(n+\frac{1}{2}\right) \alpha-x \quad \text { on }[n \alpha,(n+1) \alpha]
$$

and using Green's formula with $\phi \psi^{\alpha}$ as a test function lead to

$$
\begin{aligned}
-\int_{0}^{T} \int_{\mathcal{O}^{\alpha}} f^{\alpha} v_{x} \phi \partial_{x} \psi^{\alpha} & d x d k d t \\
= & \int_{0}^{T} \int_{\mathcal{O}^{\alpha}} f^{\alpha}\left(\alpha \partial_{t} \phi+\mathcal{A}^{\alpha} \phi\right) \psi^{\alpha} d x d k d t \\
& -\sum_{n \in \mathbb{Z}} \int_{0}^{T} \int_{B} v_{x}\left[\gamma_{(n+1) \alpha}^{-}\left(f^{\alpha} \phi \psi^{\alpha}\right)-\gamma_{n \alpha}^{+}\left(f^{\alpha} \phi \psi^{\alpha}\right)\right] d k d t
\end{aligned}
$$

Since $\left|\psi^{\alpha}\right|_{\mathcal{L}^{\infty}} \leq \alpha$, the first term of the right-hand side of (4.12), denoted by $M_{\alpha}$, can be estimated by

$$
\left|M_{\alpha}\right| \leq \alpha N(\phi)\left|f^{\alpha}\right|_{L^{2}([0, T] \times \mathbb{R} \times B)}^{2},
$$

where $N(\phi)$ depends on $\phi$ and its derivatives. Since $\partial_{x} \psi^{\alpha}=-1$ and $\gamma_{(n+1) \alpha}^{-}\left(\psi^{\alpha}\right)=$ $-\frac{\alpha}{2}, \gamma_{n \alpha}^{+}\left(\psi^{\alpha}\right)=\frac{\alpha}{2}$, we deduce from (4.12) that

$$
\left\langle v_{x} f^{\alpha}, \phi\right\rangle_{\mathcal{D}^{\prime}, \mathcal{D}}=M_{\alpha}+\frac{\alpha}{2} \sum_{n \in \mathbb{Z}} \int_{0}^{T} \int_{B} v_{x}\left[\gamma_{n \alpha}^{+}\left(f^{\alpha}\right) \phi(n \alpha)+\gamma_{(n+1) \alpha}^{-}\left(f^{\alpha}\right) \phi((n+1) \alpha)\right] d k d t .
$$

On the other hand, we have

$$
\begin{aligned}
\left\langle v_{x} \tilde{f}^{\alpha}, \phi\right\rangle_{\mathcal{D}^{\prime}, \mathcal{D}}=\sum_{n \in \mathbb{Z}} \int_{0}^{T} \int_{B} v_{x}(k)\left[\gamma_{n \alpha}^{+}\left(f^{\alpha}\right)\right. & \int_{n \alpha}^{\left(n+\frac{1}{2}\right) \alpha} \phi(x, k, t) d x \\
& \left.\quad+\gamma_{(n+1) \alpha}^{-}\left(f^{\alpha}\right) \int_{\left(n+\frac{1}{2}\right) \alpha}^{(n+1) \alpha} \phi(x, k, t) d x\right] d k d t .
\end{aligned}
$$

Taking the difference of these two identities and using the inequalities

$$
\begin{array}{r}
\sum_{n \in \mathbb{Z}} \int_{0}^{T} \int_{B} \int_{n \alpha}^{\left(n+\frac{1}{2}\right) \alpha}|\phi(n \alpha, k, t)-\phi(x, k, t)|^{2} d x d k d t \leq C(\phi) \alpha^{2}, \\
\sum_{n \in \mathbb{Z}} \int_{0}^{T} \int_{B} \int_{\left(n+\frac{1}{2}\right) \alpha}^{(n+1) \alpha}|\phi((n+1) \alpha, k, t)-\phi(x, k, t)|^{2} d x d k d t \leq C(\phi) \alpha^{2},
\end{array}
$$


we obtain, after some Cauchy-Schwarz inequalities,

$$
\begin{aligned}
\left|\left\langle v_{x}\left(f^{\alpha}-\tilde{f}^{\alpha}\right), \phi\right\rangle_{\mathcal{D}^{\prime} \mathcal{D}}\right| \leq & \alpha N(\phi)\left|f^{\alpha}\right|_{L^{2}([0, T] \times \mathbb{R} \times B)}^{2} \\
& +\alpha C(\phi)\left(\sum_{n \in \mathbb{Z}} \int_{0}^{T} \int_{K}\left|v_{x}\right|\left|\gamma_{n \alpha}^{+}\left(f^{\alpha}\right)\right|^{2} d k d t\right)^{1 / 2} \\
& +\alpha C(\phi)\left(\sum_{n \in \mathbb{Z}} \int_{0}^{T} \int_{K}\left|v_{x} \| \gamma_{n \alpha}^{-}\left(f^{\alpha}\right)\right|^{2} d k d t\right)^{1 / 2},
\end{aligned}
$$

where $K$ is a compact set containing $\{\varepsilon(k),(x, k, t) \in \operatorname{supp}(\phi)\}$. Finally, this leads to the estimate

$$
\begin{array}{r}
\left|\left\langle v_{x}\left(f^{\alpha}-\tilde{f}^{\alpha}\right), \phi\right\rangle_{\mathcal{D}^{\prime}, \mathcal{D}}\right| \leq \alpha C(\phi)\left[\left|P \gamma_{\alpha}^{\text {out }}\left(f^{\alpha}\right)\right|_{L^{2}\left(0 . T, L^{2}\left(\Gamma^{\alpha}\right)\right)}^{2}+\left|P \gamma_{\alpha}^{\text {inc }}\left(f^{\alpha}\right)\right|_{L^{2}\left(0, T, L^{2}\left(\Gamma^{\alpha}\right)\right)}^{2}\right. \\
\left.+C_{K, \delta}\left|q^{\alpha}\left(f^{\alpha}\right)\right|_{L^{2}\left(0, T, L_{\delta}^{2}\left(\Gamma^{\alpha}\right)\right)}^{2}\right]^{1 / 2}
\end{array}
$$

which shows that $f^{\alpha}-\tilde{f}^{\alpha} \rightarrow 0$ in the distributional sense.

4.3. The kinetic problem in weak form and the continuity equation. We first write problem (3.38) in weak form. Green's formula (3.23) immediately gives the following lemma.

LEmma 4.3. Let $f^{\alpha}$ be the solution of problem (3.38). Then, for any test function $\psi$ in $C^{1}\left([0, T], H_{0}^{\alpha}(\mathcal{A})\right)$ such that $\psi(\cdot, \cdot, T) \equiv 0$, and compactly supported with respect to $k$ in $\varepsilon^{-1}(\stackrel{\circ}{\mathcal{R}})$, we have

$$
\begin{array}{r}
\int_{0}^{T} \int_{\mathcal{O}^{\alpha}} f^{\alpha}\left(\alpha \partial_{t} \psi+v_{x}(k) \partial_{x} \psi+\partial_{x} V \partial_{k_{x}} \psi\right) d x d k d t+\left.\alpha \int_{\mathcal{O}^{\alpha}} f_{I}^{\alpha} \psi\right|_{t=0} d x d k \\
=\frac{1}{\alpha} \int_{0}^{T}\left(\gamma_{\alpha}^{\text {out }}\left(f^{\alpha}(t)\right), \gamma_{\alpha}^{\text {out }}(\psi(t))-\mathcal{B}^{\alpha *} \gamma_{\alpha}^{\text {inc }}(\psi(t))\right)_{\Gamma^{\alpha}} d t
\end{array}
$$

The classical structure of a diffusion approximation problem can now be clearly seen on (4.13), since the interface scattering operator appears explicitly, multiplied by the right scaling factor $1 / \alpha$ (see, e.g., [31], [8]). We now define the density and current of particles of energy $\varepsilon$ by

$$
\begin{aligned}
F^{\alpha}(x, \varepsilon, t) & =\frac{1}{N(\varepsilon)} \int_{B} f^{\alpha}(x, k, t) \delta(\varepsilon(k)-\varepsilon) d k \\
J^{\alpha}(x, \varepsilon, t) & =\frac{1}{\alpha} \int_{B} f^{\alpha}(x, k, t) v_{x}(k) \delta(\varepsilon(k)-\varepsilon) d k .
\end{aligned}
$$

We have

Lemma 4.4. For any test function $\phi(x, \varepsilon, t)$ in $C_{c}^{1}([0, T] \times \mathbb{R} \times \mathcal{R})$ (i.e., continuously differentiable and compactly supported in $[0, T] \times \mathbb{R} \times \mathcal{R})$, such that $\phi(\cdot, \cdot, T) \equiv 0$, we have

$$
\int_{0}^{T} \int_{\Omega^{\alpha} \times \mathcal{R}}\left[N(\varepsilon) F^{\alpha} \partial_{t} \phi+J^{\alpha}\left(\partial_{x} \phi+\partial_{x} V \partial_{\varepsilon} \phi\right)\right] d x d \varepsilon d t+\left.\int_{\mathbb{R} \times \mathcal{R}} F_{I} \phi\right|_{t=0} d x d k=0 .
$$


Proof. When $\phi$ is compactly supported in $\stackrel{\circ}{\mathcal{R}}$ with respect to $\varepsilon$, this lemma obviously results from the weak formulation (4.13) with $\phi$ as a test function $\psi$. For $\phi$ compactly supported in $\mathcal{R}$, we cannot use Green's formula as in Lemma 4.3 and we have to resort to the approximate solution $f_{\eta}^{\alpha}$, for which Green's formula $(3.23)$ can be applied. We have

$$
\begin{aligned}
\int_{0}^{T} & \int_{\mathcal{O}^{\alpha}} f_{\eta}^{\alpha}\left(\alpha \partial_{t} \phi+v_{x}(k) \partial_{x} \phi+\partial_{x} V \partial_{k_{x}} \phi\right) d x d k d t+\left.\alpha \int_{\mathcal{O}^{\alpha}} F_{\eta} \phi\right|_{t=0} d x d k \\
& =\frac{1}{\alpha} \int_{0}^{T}\left[\left(\gamma_{\alpha}^{\text {out }}\left(f_{\eta}^{\alpha}(t)\right), \gamma_{\alpha}^{\text {out }}(\phi(t))\right)_{\Gamma^{\alpha}}-\left(\mathcal{B}_{\eta} \gamma_{\alpha}^{\text {out }}\left(f_{\eta}^{\alpha}(t)\right), \gamma_{\alpha}^{\text {inc }}(\phi(t))\right)_{\Gamma^{\alpha}}\right] d t \\
& =\frac{1}{\alpha} \int_{0}^{T}\left(Q \gamma_{\alpha}^{\text {out }}\left(f_{\eta}^{\alpha}(t)\right)-Q \mathcal{B}_{\eta} \gamma_{\alpha}^{\text {out }}\left(f_{\eta}^{\alpha}(t)\right), \gamma_{\alpha}(\phi(t))\right)_{\Gamma^{\alpha}} d t
\end{aligned}
$$

where $\gamma_{\alpha}(\phi):=\gamma_{\alpha}^{\text {inc }}(\phi)=\gamma_{\alpha}^{\text {out }}(\phi)$. Thanks to the co-area formula, and to the orthogonality between $P \gamma_{\alpha}^{\text {out }}\left(f_{\eta}^{\alpha}\right)$ and $\gamma(\phi)$, this leads to

$$
\begin{array}{r}
\int_{0}^{T} \int_{\Omega^{\alpha} \times \mathcal{R}}\left[N(\varepsilon) F_{\eta}^{\alpha} \partial_{t} \phi+J_{\eta}^{\alpha}\left(\partial_{x} \phi+\partial_{x} V \partial_{\varepsilon} \phi\right)\right] d x d \varepsilon d t+\left.\int_{\mathbb{R} \times \mathcal{R}} F_{\eta} \phi\right|_{t=0} d x d k \\
=\frac{1}{\alpha} \int_{0}^{T}\left(\gamma_{\alpha}^{\text {out }}\left(f_{\eta}^{\alpha}(t)\right),\left(1-\frac{1}{1+\eta}\right) \gamma(\phi(t))\right)_{\Gamma^{\alpha}} d t
\end{array}
$$

For $\eta$ small enough, the computation (3.35) gives the inequality

$$
\left(1-\frac{1}{(1+\eta)^{2}}\right)\left|\gamma_{\alpha}^{\text {out }}\left(f_{\eta}^{\alpha}(t)\right)\right|_{L^{2}\left(\Gamma^{\alpha}\right)}^{2} \leq 2 \alpha\left(\mathcal{A}^{\alpha} f_{\eta}^{\alpha}, f_{\eta}^{\alpha}\right) \leq 2 \alpha\left|F_{I}\right|_{H^{\alpha}(\mathcal{A}, \mathcal{B})}^{2} .
$$

Thus, we have

$$
\left(1-\frac{1}{1+\eta}\right)\left|\gamma_{\alpha}^{\text {out }}\left(f_{\eta}^{\alpha}(t)\right)\right|_{L^{2}\left(\Gamma^{\alpha}\right)} \rightarrow 0
$$

as $\eta \rightarrow 0$. Therefore, the right-hand side of (4.17) tends to 0 as $\eta \rightarrow 0$, which concludes the proof.

We are now aiming at taking the limit $\alpha \rightarrow 0$. We obviously have

$$
F^{\alpha} \rightarrow \frac{1}{N(\varepsilon)} \int_{B} f^{0}(x, k, t) \delta(\varepsilon(k)-\varepsilon) d k=F,
$$

in $L^{\infty}\left(0, T, L^{2}(\mathbb{R} \times \mathcal{R})\right)$ weak star. Note that $J^{\alpha}$ is the ratio of two quantities tending to zero as $\alpha \rightarrow 0$, because $\int_{\mathcal{S}_{\varepsilon}} v_{x}(k) F d N_{\varepsilon}(k)=0$. In the next section, we prove that $J^{\alpha}$ has a finite limit.

4.4. Existence of a limit for the current. $J^{\alpha}$ is defined on $\Omega^{\alpha} \times \mathcal{R}$ and has traces $\gamma_{n \alpha}^{ \pm}\left(J^{\alpha}\right)$ at the interfaces, obviously given by

$$
\gamma_{n \alpha}^{ \pm}\left(J^{\alpha}\right)(\varepsilon, t)=\frac{1}{\alpha} \int_{B} \gamma_{n \alpha}^{ \pm}\left(f^{\alpha}\right)(k, t) v_{x}(k) \delta(\varepsilon(k)-\varepsilon) d k .
$$

By the current conservation assumption (3.5), it is readily seen that

$$
\gamma_{n \alpha}^{+}\left(J^{\alpha}\right)(\varepsilon, t)=\gamma_{n \alpha}^{-}\left(J^{\alpha}\right)(\varepsilon, t):=J_{n \alpha}^{\alpha}(\varepsilon, t),
$$

thus defining the quantity $J_{n \alpha}^{\alpha}$. 
From this quantity, we construct the piecewise constant function $\widetilde{J}^{\alpha}$ as follows:

$$
\widetilde{J}^{\alpha}(x, \varepsilon, t)=J_{n \alpha}^{\alpha}(\varepsilon, t), \quad x \in\left[\left(n-\frac{1}{2}\right) \alpha,\left(n+\frac{1}{2}\right) \alpha\right] .
$$

The existence of a limit of $J^{\alpha}$ is obtained through that of $\widetilde{J}^{\alpha}$ as the following lemma states.

LEMma $4.5 . \widetilde{J}^{\alpha}$ is bounded in $L^{2}([0, T] \times \mathbb{R} \times \mathcal{R})$ and we have

$$
J^{\alpha}-\widetilde{J}^{\alpha} \rightarrow 0
$$

as $\alpha \rightarrow 0$, in the distributional sense. There exists $J$ in $L^{2}([0, T] \times \mathbb{R} \times \mathcal{R})$ such that, up to the extraction of a subsequence,

$$
\tilde{J}^{\alpha} \rightarrow J \text { in } L^{2}([0, T] \times \mathbb{R} \times \mathcal{R}) \text { weak }
$$

and $J^{\alpha} \rightarrow J$ in the distributional sense and, more precisely,

$$
\int_{0}^{T} \int_{\mathcal{O}^{\alpha} \times \mathcal{R}} J^{\alpha} \varphi d x d \varepsilon d t \rightarrow \int_{0}^{T} \int_{\mathbb{R} \times \mathcal{R}} J \varphi d x d \varepsilon d t
$$

as $\alpha \rightarrow 0$ for all test functions $\varphi$ in $C_{c}^{1}([0, T] \times \mathbb{R} \times \mathcal{R})$.

We note that the convergence (4.23) allows us to pass to the limit in (4.16) and to obtain

Corollary 4.6. For any test function $\phi(x, \varepsilon, t)$ in $C_{c}^{2}([0, T] \times \mathbb{R} \times \mathcal{R})$ (twice continuously differentiable with compact support in $\mathbb{R} \times \mathcal{R}$ ) such that $\phi(\cdot, \cdot, T) \equiv 0$, we have

$$
\int_{0}^{T} \int_{\Omega^{\alpha} \times \mathcal{R}}\left[N(\varepsilon) F \partial_{t} \phi+J\left(\partial_{x} \phi+\partial_{x} V \partial_{\varepsilon} \phi\right)\right] d x d \varepsilon d t+\left.\int_{\mathbb{R} \times \mathcal{R}} F_{I} \phi\right|_{t=0} d x d k=0
$$

Equation (4.16) is nothing else but the weak form of the continuity equation (2.13) with initial condition (2.15). Note that we obtain the zero flux boundary condition (2.16) by means of an integration by parts in (4.24).

Proof of Lemma 4.5. We first define the current carried by the outgoing and incoming traces:

$$
\begin{aligned}
J_{n \alpha}^{\alpha, \text { out }}(\varepsilon, t) & =\frac{1}{\alpha} \int_{B} \gamma_{n \alpha}^{\text {out }}\left(f^{\alpha}\right)(k, t) v_{x}(k) \delta(\varepsilon(k)-\varepsilon) d k, \\
J_{n \alpha}^{\alpha, \text { inc }}(\varepsilon, t) & =\frac{1}{\alpha} \int_{B} \gamma_{n \alpha}^{\text {inc }}\left(f^{\alpha}\right)(k, t) v_{x}(k) \delta(\varepsilon(k)-\varepsilon) d k .
\end{aligned}
$$

From these quantities, we construct the piecewise constant functions $J^{\alpha, \text { out }}$ and $J^{\alpha, \text { inc }}$ as follows:

$$
\left\{\begin{array}{l}
J^{\alpha, \text { out }}(x, \varepsilon, t)=J_{n \alpha}^{\alpha, \text { out }}(\varepsilon, t) \\
J^{\alpha, \text { inc }}(x, \varepsilon, t)=J_{n \alpha}^{\alpha, \text { inc }}(\varepsilon, t)
\end{array}\right\}, \quad x \in\left[\left(n-\frac{1}{2}\right) \alpha,\left(n+\frac{1}{2}\right) \alpha\right] .
$$

These various definitions are connected via the relation (obvious from definitions (2.3) and (2.2)):

$$
\gamma_{n \alpha}^{\text {out }}\left(f^{\alpha}\right)+\gamma_{n \alpha}^{\text {inc }}\left(f^{\alpha}\right)=\gamma_{n \alpha}^{+}\left(f^{\alpha}\right)+\gamma_{n \alpha}^{-}\left(f^{\alpha}\right)
$$


which shows that

$$
J_{n \alpha}^{\alpha}=\gamma_{n \alpha}^{+}\left(J^{\alpha}\right)=\gamma_{n \alpha}^{-}\left(J^{\alpha}\right)=\frac{1}{2}\left(\gamma_{n \alpha}^{+}\left(J^{\alpha}\right)+\gamma_{n \alpha}^{-}\left(J^{\alpha}\right)\right)=\frac{1}{2}\left(J_{n \alpha}^{\alpha, \text { out }}+J_{n \alpha}^{\alpha, \text { inc }}\right) .
$$

By (4.25), in order to prove that $\widetilde{J}^{\alpha}$ is bounded in $L^{2}([0, T] \times \mathbb{R} \times \mathcal{R})$, it is enough to prove that $J^{\alpha, \text { out }}$ and $J^{\alpha \text {,inc }}$ separately are bounded in this space. We prove it for $J^{\alpha, \text { out }}$, the proof being similar for $J^{\alpha, \text { inc }}$. Noting that

$$
\int_{\mathcal{S}_{\varepsilon}} \gamma_{n \alpha}^{\text {out }}\left(f^{\alpha}\right) v_{x}(k) d N_{\varepsilon}(k)=\int_{\mathcal{S}_{\varepsilon}} P \gamma_{n \alpha}^{\text {out }}\left(f^{\alpha}\right) v_{x}(k) d N_{\varepsilon}(k),
$$

we have

$$
\begin{aligned}
\left|J^{\alpha, \text { out }}\right|_{L^{2}([0, T] \times \mathbb{R} \times \mathcal{R})}^{2} & =\alpha \int_{0}^{T} \sum_{n \in \mathbb{Z}} \int_{\mathcal{R}}\left|J_{n \alpha}^{\alpha, \text { out }}(\varepsilon, t)\right|^{2} d \varepsilon d t \\
& =\alpha \int_{0}^{T} \sum_{n \in \mathbb{Z}} \int_{\mathcal{R}}\left|\frac{1}{\alpha} \int_{\mathcal{S}_{\varepsilon}} P\left(\gamma_{n \alpha}^{\text {out }}\left(f^{\alpha}\right)\right) v_{x}(k) d N_{\varepsilon}(k)\right|^{2} d \varepsilon d t .
\end{aligned}
$$

Then, using the Cauchy-Schwarz inequality and (4.4), we get

$$
\begin{aligned}
\left|J^{\alpha, \text { out }}\right|_{L^{2}([0, T] \times \mathbb{R} \times \mathcal{R})}^{2} & \leq C \frac{1}{\alpha} \int_{0}^{T} \sum_{n \in \mathbb{Z}} \int_{\mathcal{R}} \int_{\mathcal{S}_{\varepsilon}}\left|P \gamma_{n \alpha}^{\text {out }}\left(f^{\alpha}\right)\right|^{2}\left|v_{x}(k)\right| d N_{\varepsilon}(k) d \varepsilon d t \\
& \leq C \frac{1}{\alpha} \int_{0}^{T} \sum_{n \in \mathbb{Z}}\left|P \gamma_{n \alpha}^{\text {out }}\left(f^{\alpha}\right)\right|_{L^{2}(B)}^{2} d t \leq C\left|F_{I}\right|_{L^{2}(\mathbb{R} \times \mathcal{R})}^{2},
\end{aligned}
$$

which proves the first statement of Lemma 4.5.

We now prove (4.21). Let $\varphi$ be a test function in $\mathcal{D}(] 0, T[\times \mathbb{R} \times \mathcal{R})$. We proceed as in the proof of Lemma 4.2: we introduce

$$
\psi^{\alpha}(x)=\left(n+\frac{1}{2}\right) \alpha-x \quad \text { on }[n \alpha,(n+1) \alpha]
$$

and the quantity

$$
M_{\alpha}^{\prime}=\frac{1}{\alpha} \int_{0}^{T} \int_{\mathcal{O}^{\alpha}} f^{\alpha}\left(\alpha \partial_{t} \varphi+\mathcal{A}^{\alpha} \varphi\right) \psi^{\alpha} d x d k d t .
$$

The first step of the proof consists in establishing the equality

$$
\begin{aligned}
M_{\alpha}^{\prime}= & \int_{0}^{T} \int_{\Omega^{\alpha}} \int_{\mathcal{R}} J^{\alpha} \varphi d \varepsilon d x d t \\
& -\frac{\alpha}{2} \sum_{n \in \mathbb{Z}} \int_{0}^{T} \int_{\mathcal{R}}\left[\varphi(n \alpha) J_{n \alpha}^{\alpha}+\varphi((n+1) \alpha) J_{(n+1) \alpha}^{\alpha}\right] d \varepsilon d t .
\end{aligned}
$$

Since we cannot apply Green's formula because $\varphi$ is compactly supported in $\mathcal{R}$ but not in $\stackrel{\circ}{\mathcal{R}}$, we use the approximate solution $f_{\eta}^{\alpha}$ once more. Let $M_{\eta}$ be defined by

$$
M_{\eta}=\frac{1}{\alpha} \int_{0}^{T} \int_{\mathcal{O}^{\alpha}} f_{\eta}^{\alpha}\left(\alpha \partial_{t} \varphi+\mathcal{A}^{\alpha} \varphi\right) \psi^{\alpha} d x d k d t
$$


We have $\mathcal{A}^{\alpha}\left(\psi^{\alpha}\right)=-v_{x}(k)$ and $\gamma_{n \alpha}^{ \pm}\left(\psi^{\alpha}\right)= \pm \frac{\alpha}{2}$. Then, Green's formula gives

$$
\begin{aligned}
M_{\eta}= & \int_{0}^{T} \int_{\Omega^{\alpha}} \int_{\mathcal{R}} J_{\eta}^{\alpha} \varphi d \varepsilon d x d t \\
& -\frac{\alpha}{2} \sum_{n \in \mathbb{Z}} \int_{0}^{T} \int_{\mathcal{R}}\left[\varphi(n \alpha) J_{\eta, n \alpha}^{+}+\varphi((n+1) \alpha) J_{\eta,(n+1) \alpha}^{-}\right] d \varepsilon d t,
\end{aligned}
$$

where

We have

$$
J_{\eta, n \alpha}^{ \pm}=\frac{1}{\alpha} \int_{B} \gamma_{n \alpha}^{ \pm}\left(f_{\eta}^{\alpha}\right)(k, t) v_{x}(k) \delta(\varepsilon(k)-\varepsilon) d k .
$$

$$
\begin{aligned}
\int_{B} \gamma_{n \alpha}^{+}\left(f_{\eta}^{\alpha}\right)(k, t) v_{x}(k) \delta(\varepsilon(k)-\varepsilon) d k \\
=\int_{v_{x}(k)<0} \gamma_{n \alpha}^{\text {out }}\left(f_{\eta}^{\alpha}\right) v_{x} \delta(\varepsilon(k)-\varepsilon) d k+\int_{v_{x}(k)>0} \gamma_{n \alpha}^{\text {inc }}\left(f_{\eta}^{\alpha}\right) v_{x} \delta(\varepsilon(k)-\varepsilon) d k \\
=\int_{v_{x}(k)<0} P \gamma_{n \alpha}^{\text {out }}\left(f_{\eta}^{\alpha}\right) v_{x} \delta(\varepsilon(k)-\varepsilon) d k+\int_{v_{x}(k)>0} P \gamma_{n \alpha}^{\text {inc }}\left(f_{\eta}^{\alpha}\right) v_{x} \delta(\varepsilon(k)-\varepsilon) d k \\
\quad+\left(\frac{1}{1+\eta}-1\right) \int_{v_{x}(k)>0} Q \gamma_{n \alpha}^{\text {out }}\left(f_{\eta}^{\alpha}\right)\left|v_{x}\right| \delta(\varepsilon(k)-\varepsilon) d k
\end{aligned}
$$

The last term vanishes when $\eta$ goes to 0 , thanks to (4.18). We deduce that the sequence $\left(J_{\eta, n \alpha}^{ \pm}\right)_{n \in \mathbb{Z}}$ is bounded in $L^{2}\left(\Gamma^{\alpha}\right)$ and that it converges to the sequence $\left(J_{n \alpha}^{\alpha}\right)_{n \in \mathbb{Z}}$ weakly in $L^{2}\left(\Gamma^{\alpha}\right)$. That $J_{\eta}^{\alpha}$ converges to $J^{\alpha}$ in $L^{2}([0, T] \times \mathbb{R} \times \mathcal{R})$ weakly follows from Proposition 3.11. It is now readily seen that $(4.29)$ holds. Now, we write

$$
\begin{aligned}
\left\langle\left(J^{\alpha}-\widetilde{J}^{\alpha}\right), \varphi\right\rangle_{\mathcal{D}^{\prime}, \mathcal{D}} & \\
= & \int_{0}^{T} \int_{\Omega^{\alpha}} \int_{\mathcal{R}} J^{\alpha} \varphi(x, \varepsilon, t) d \varepsilon d x d t-\int_{0}^{T} \int_{\Omega^{\alpha}} \int_{\mathcal{R}} \widetilde{J}^{\alpha}(x, \varepsilon, t) \varphi(x, \varepsilon, t) d \varepsilon d x d t \\
= & M_{\alpha}^{\prime}+\sum_{n \in \mathbb{Z}} \int_{0}^{T} \int_{\mathcal{R}}\left[J_{n \alpha}^{\alpha}(\varepsilon, t) \int_{n \alpha}^{\left(n+\frac{1}{2}\right) \alpha}[\varphi(n \alpha, \varepsilon, t)-\varphi(x, \varepsilon, t)] d x\right. \\
& \left.+J_{(n+1) \alpha}^{\alpha}(\varepsilon, t) \int_{\left(n+\frac{1}{2}\right) \alpha}^{(n+1) \alpha}[\varphi((n+1) \alpha, \varepsilon, t)-\varphi(x, \varepsilon, t)] d x\right] d \varepsilon d t .
\end{aligned}
$$

In the same way as in Lemma 4.2 , we have

$$
\begin{aligned}
& \sum_{n \in \mathbb{Z}} \int_{0}^{T} \int_{\mathcal{R}} J_{n \alpha}^{\alpha}(\varepsilon, t) \int_{n \alpha}^{\left(n+\frac{1}{2}\right) \alpha}[\varphi(n \alpha, \varepsilon, t)-\varphi(x, \varepsilon, t)] d x d \varepsilon \\
& \quad \leq C\left[\left|J_{n \alpha}^{\alpha, \text { out }}\right|_{L^{2}\left(0, T, L^{2}\left(\Gamma^{\alpha}\right)\right)}^{2}+\left|J_{n \alpha}^{\alpha, \text { inc }}\right|_{L^{2}\left(0, T, L^{2}\left(\Gamma^{\alpha}\right)\right)}^{2}\right]^{1 / 2} N(\varphi) \alpha \leq C \alpha,
\end{aligned}
$$

where $N(\varphi)$ denotes generic continuous semi-norms on the space of infinitely differentiable functions $\mathcal{D}$, applied to $\varphi$. A similar estimate holds for the term involving $J_{(n+1) \alpha}^{\alpha}$.

The remainder will be devoted to the proof that $M_{\alpha}^{\prime}=\mathcal{O}(\alpha)$ as $\alpha \rightarrow 0$. First, we write, since $\left|\psi^{\alpha}\right|_{\infty}=\frac{\alpha}{2}$,

$$
\left|M_{\alpha}^{\prime}\right| \leq N(\varphi) \alpha+\left|\frac{1}{\alpha} \int_{0}^{T} \int_{\mathcal{O}^{\alpha}} f^{\alpha}\left(\mathcal{A}^{\alpha} \varphi\right) \psi^{\alpha} d x d \varepsilon d t\right|
$$


We denote by $H^{\alpha}$ the last term in (4.30), and we apply (4.28) with $\partial_{x} \varphi+\partial_{x} V \partial_{\varepsilon} \varphi=\varphi_{0}$ instead of $\varphi$, and the function $\mu^{\alpha}$ instead of $\psi^{\alpha}$, where $\mu^{\alpha}$ is a primitive of $\psi^{\alpha}$ given by

$$
\mu^{\alpha}(x)=-\frac{1}{2}\left(\left(n+\frac{1}{2}\right) \alpha-x\right)^{2} \quad \text { on }[n \alpha,(n+1) \alpha] .
$$

We obtain

$$
\begin{aligned}
M_{\alpha}^{\prime \prime}= & \frac{1}{\alpha} \int_{0}^{T} \int_{\mathcal{O}^{\alpha}} f^{\alpha}\left(\alpha \partial_{t} \varphi_{0}+\mathcal{A}^{\alpha} \varphi_{0}\right) \mu^{\alpha} d x d k d t \\
= & -\int_{0}^{T} \int_{\Omega^{\alpha} \times \mathcal{R}} J^{\alpha} \varphi_{0} \psi^{\alpha} d x d k d t \\
& -\sum_{n \in \mathbb{Z}} \int_{0}^{T} \int_{\mathcal{R}}\left[\varphi_{0}(n \alpha) J_{n \alpha}^{\alpha} \mu^{\alpha}(n \alpha)-\varphi_{0}((n+1) \alpha) J_{(n+1) \alpha}^{\alpha} \mu^{\alpha}((n+1) \alpha)\right] d \varepsilon d t
\end{aligned}
$$

where, like for (4.29), the identity must first be proven with $f_{\eta}^{\alpha}$, followed by the limit $\eta \rightarrow 0$. The details are left to the reader. Equation (4.32) gives

$$
\begin{aligned}
\left|H^{\alpha}\right|= & \left|\frac{1}{\alpha} \int_{0}^{T} \int_{\mathcal{O}^{\alpha}} f^{\alpha} v_{x} \varphi_{0} \psi^{\alpha} d x d k d t\right| \\
\leq & \left|M_{\alpha}^{\prime \prime}\right|+\sum_{n \in \mathbb{Z}} \int_{0}^{T} \int_{\mathcal{R}}\left\{\left|\varphi_{0}(n \alpha)\right|\left|J_{n \alpha}^{\alpha}\right|\left|\mu^{\alpha}(n \alpha)\right|\right. \\
& \left.+\left|\varphi_{0}((n+1) \alpha)\right|\left|J_{(n+1) \alpha}^{\alpha}\right|\left|\mu^{\alpha}((n+1) \alpha)\right|\right\} d t d \varepsilon
\end{aligned}
$$

and, since $\left|\mu^{\alpha}\right|_{L^{\infty}} \leq C \alpha^{2}$,

$$
\begin{aligned}
\sum_{n \in \mathbb{Z}} & \int_{0}^{T} \int_{\mathcal{R}}\left|\varphi_{0}(n \alpha)\right|\left|J_{n \alpha}^{\alpha}\right|\left|\mu^{\alpha}(n \alpha)\right| d \varepsilon \\
& \leq C \alpha \int_{0}^{T} \int_{\mathcal{R}} \alpha \sum_{n \in \mathbb{Z}}\left|\varphi_{0}(n \alpha)\right|\left|J_{n \alpha}^{\alpha}\right| d \varepsilon d t \\
& \leq C \alpha\left(\int_{0}^{T} \int_{\mathcal{R}} \alpha \sum_{n \in \mathbb{Z}}\left|\varphi_{0}(n \alpha)\right|^{2} d \varepsilon d t\right)^{1 / 2}\left(\int_{0}^{T} \int_{\mathcal{R}} \alpha \sum_{n \in \mathbb{Z}}\left|J_{n \alpha}^{\alpha}\right|^{2} d \varepsilon d t\right)^{1 / 2} \\
& \leq \alpha N(\varphi)\left|\widetilde{J}^{\alpha}\right|_{L^{2}([0, T[\times \mathbb{R} \times \mathcal{R}) .}
\end{aligned}
$$

Moreover, we deduce from (4.31) that

$$
\left|M_{\alpha}^{\prime \prime}\right| \leq N(\varphi) \alpha
$$

This gives that $H^{\alpha}=\mathcal{O}(\alpha)$, and the result follows.

The aim of the next section is to derive Eq. (2.14) for the current.

4.5. Equation for the current. We are first concerned with the auxiliary equation (2.18), (2.19). We prove

Lemma 4.7. The solution $\chi$ of problem (2.18), (2.19) exists and is unique. Moreover, $\chi$ and $\partial_{x} \chi$ belong to $L^{2}\left(\mathcal{S}_{\varepsilon}\right)$, uniformly for $(x, \varepsilon) \in \mathbb{R} \times \mathcal{R}$ (i.e., $\chi$ and $\partial_{x} \chi$ belong to $\left.L^{\infty}\left(\mathbb{R} \times \mathcal{R}, L^{2}\left(\mathcal{S}_{\varepsilon}\right)\right)\right)$. 
Proof. We consider Eq. (2.18) as a functional equation for functions $\chi \in L^{2}\left(\mathcal{S}_{\varepsilon}\right)$, parametrized by $(x, \varepsilon) \in \mathbb{R} \times \mathcal{R}$. With Hypothesis $3.3, I-\mathcal{B}^{*}$ is a Fredholm operator. Thus, with $(3.12), R\left(I-\mathcal{B}^{*}\right)=N(I-\mathcal{B})^{\perp}=\operatorname{Span}(1)^{\perp}$, where $R$ and $N$ respectively denote the Range and Null-Space. But

$$
\left(I+\mathcal{B}^{*}\right)\left(\operatorname{sgn}\left(v_{x}\right)\right)=-\left(I-\mathcal{B}^{*}\right)\left(\operatorname{sgn}\left(v_{x}\right)\right)+2\left(\operatorname{sgn}\left(v_{x}\right)\right),
$$

and it is enough to prove that $\operatorname{sgn}\left(v_{x}\right) \in R\left(I-\mathcal{B}^{*}\right)$ to deduce the existence of a solution of (2.18). We have, by the co-area formula,

$$
\begin{aligned}
\left(\operatorname{sgn}\left(v_{x}\right), 1\right)_{L^{2}\left(\mathcal{S}_{\varepsilon}\right)} & =\int_{\mathcal{S}_{\varepsilon}} v_{x}(k) d N_{\varepsilon}(k)=\int_{B} \partial_{k_{x}} \varepsilon(k) \delta(\varepsilon(k)-\varepsilon) d k \\
& =\int_{B} \partial_{k_{x}}(H(\varepsilon(k)-\varepsilon)) d k=0,
\end{aligned}
$$

because $H(\varepsilon(k)-\varepsilon$ ) (where $H$ denotes the Heaviside function) is a periodic function of $k$ and the integral over a period of the derivative of a periodic function is zero. This proves the existence of a solution of (2.18). Two such solutions may differ by an element of $N\left(I-\mathcal{B}^{*}\right)=\operatorname{Span}(1)$, i.e., a constant. It is easy to see that condition (2.19) singles out a unique solution.

Now, we prove the estimate

$$
|\chi|_{L^{2}\left(\mathcal{S}_{\varepsilon}\right)} \leq C\left|\operatorname{sgn}\left(v_{x}\right)\right|_{L^{2}\left(\mathcal{S}_{\varepsilon}\right)},
$$

where $C$ is uniform with respect to $(x, \varepsilon) \in \mathbb{R} \times \mathcal{R}$. We write

$$
\left(\left(I-\mathcal{B}^{*}\right) \chi, \chi\right)_{L^{2}\left(\mathcal{S}_{\varepsilon}\right)}=\frac{1}{2}\left(\left(I+\mathcal{B}^{*}\right) \operatorname{sgn}\left(v_{x}\right), \chi\right)_{L^{2}\left(\mathcal{S}_{\varepsilon}\right)} \leq\left|\operatorname{sgn}\left(v_{x}\right)\right|_{L^{2}\left(\mathcal{S}_{\varepsilon}\right)}|\chi|_{L^{2}\left(\mathcal{S}_{\varepsilon}\right)},
$$

and, using that $\chi \perp N(I-\mathcal{B})$ and (3.18):

$$
\begin{aligned}
\left(\left(I-\mathcal{B}^{*}\right) \chi, \chi\right)_{L^{2}\left(\mathcal{S}_{\varepsilon}\right)} & =(\chi,(I-\mathcal{B}) \chi)_{L^{2}\left(\mathcal{S}_{\varepsilon}\right)} \\
& \geq|\chi|_{L^{2}\left(\mathcal{S}_{\varepsilon}\right)}\left(|\chi|_{L^{2}\left(\mathcal{S}_{\varepsilon}\right)}-|\mathcal{B} \chi|_{L^{2}(\mathcal{S} \varepsilon)}\right) \\
& \geq(1-K)|\chi|_{L^{2}\left(\mathcal{S}_{\varepsilon}\right)}^{2},
\end{aligned}
$$

where $K<1$ is the constant appearing in (3.18), uniform with respect to $(x, \varepsilon) \in \mathbb{R} \times \mathcal{R}$. Then, (4.34) follows with $C=(1-K)^{-1}$.

We now prove a similar estimate (4.34) for $\partial_{x} \chi . \partial_{x} \chi$ is the solution of problem

$$
\left(I-\mathcal{B}^{*}\right)\left(\partial_{x} \chi\right)=\partial_{x} \mathcal{B}^{*}(\chi)+\frac{1}{2} \partial_{x} \mathcal{B}^{*}\left(\operatorname{sgn}\left(v_{x}\right)\right), \quad \partial_{x} \chi \perp N(I-\mathcal{B}) .
$$

Then, from the proof of (4.34) and the fact that $\partial_{x} \mathcal{B}^{*}$ is a bounded operator on $L^{2}\left(\mathcal{S}_{\varepsilon}\right)$, uniformly with respect to $(x, \varepsilon) \in \mathbb{R} \times \mathcal{R}$ (Hypothesis 3.4 ), it follows that

$$
\left|\partial_{x} \chi\right|_{L^{2}\left(\mathcal{S}_{\varepsilon}\right)} \leq C|\chi|_{L^{2}\left(\mathcal{S}_{\varepsilon}\right)}+C^{\prime}\left|\operatorname{sgn}\left(v_{x}\right)\right|_{L^{2}\left(\mathcal{S}_{\varepsilon}\right)} \leq C,
$$

uniformly for $(x, \varepsilon) \in \mathbb{R} \times \mathcal{R}$.

The energy variable $\varepsilon$ is also a parameter, but it appears that estimates for $\partial_{\varepsilon} \chi$ are not easily available for $\varepsilon$ close to the critical values of the function $\varepsilon(k)$. Indeed, the derivative of $\mathcal{B}_{\chi}$ with respect to $\varepsilon$ involves a term arising from the $\varepsilon$-dependence of the integration domain $\mathcal{S}_{\varepsilon}$ of the integral operator. This term may be singular in the neighbourhood of the critical values of the function $\varepsilon(k)$.

We will now establish the current equation. 
LEMma 4.8. $F$ and $J$ satisfy the current equation $(2.14)$ in the space of $L^{2}(\mathbb{R} \times \mathcal{R})$.

Proof. In order to derive the current equation (2.14), we wish to use the weak formulation (4.13) with $\chi(x, k) \phi(x, \varepsilon(k), t)$ as a test function $\psi$. However, although $\chi$ is regular with respect to $x$ as guaranteed by Lemma 4.7, it may be nonregular with respect to $k$. We can bypass this problem by assuming that the following property holds.

Hypothesis 4.1. $\chi$ belongs to $\mathcal{C}^{0}\left(\mathbb{R} \times \stackrel{\circ}{\mathcal{R}}, L^{2}\left(\mathcal{S}_{\varepsilon}\right)\right)$. Therefore, there exists a sequence of regularized functions $\chi_{\rho}$ in $\mathcal{C}_{c}^{\infty}(\mathbb{R} \times B)$ such that

$$
\chi_{\rho} \rightarrow \chi \quad \text { in } L_{\text {loc }}^{\infty}\left(\mathbb{R} \times \stackrel{\circ}{\mathcal{R}}, L^{2}\left(\mathcal{S}_{\varepsilon}\right)\right)
$$

Note that the only restrictive hypothesis concerns the regularity with respect to $\varepsilon$, since the regularity with respect to $x$ is guaranteed by Lemma 4.7. We shall check in the last section that Hypothesis 4.1 is not empty, and is fulfilled at least in some particular cases.

We insert $\psi=\chi_{\rho} \phi$ as a test function in (4.13). We obtain

$$
\begin{aligned}
\frac{1}{\alpha} \int_{0}^{T} & \alpha \sum_{n \in \mathbb{Z}} \int_{B} \gamma_{n \alpha}^{\text {out }}\left(f^{\alpha}\right)(k, t) \\
& \times\left(\left(I-\mathcal{B}(n \alpha)^{*}\right) \chi_{\rho}(n \alpha, k)\right) \phi(n \alpha, \varepsilon(k), t)\left|v_{x}(k)\right| d k d x d t \\
= & \alpha \int_{0}^{T} \int_{\mathcal{O}^{\alpha}} f^{\alpha} \chi_{\rho} \partial_{t} \phi d x d k d t+\left.\alpha \int_{\mathcal{O}^{\alpha}} f_{I}^{\alpha} \chi_{\rho} \phi\right|_{t=0} d x d k \\
& +\int_{0}^{T} \int_{\Omega^{\alpha} \times \mathcal{R}}\left(\int_{\mathcal{S}_{\varepsilon}} f^{\alpha}\left(v_{x}(k) \partial_{x}+\partial_{x} V \partial_{k_{x}}\right)\left(\phi \chi_{\rho}\right) d N_{\varepsilon}(k)\right) d x d \varepsilon d t .
\end{aligned}
$$

We first consider the right-hand side of (4.35). The first two terms are multiplied by $\alpha$ and obviously tend to zero. We take the limit $\alpha \rightarrow 0$ in the third term. Considering that both $F=\lim f^{\alpha}$ and $\phi$ only depend on $\varepsilon(k)$, we obtain

$$
\begin{aligned}
\int_{0}^{T} \int_{\Omega^{\alpha} \times \mathcal{R}}\left(\int_{\mathcal{S}_{\varepsilon}}\right. & \left.f^{\alpha}\left(v_{x}(k) \partial_{x}+\partial_{x} V \partial_{k_{x}}\right)\left(\phi \chi_{\rho}\right) d N_{\varepsilon}(k)\right) d x d \varepsilon d t \\
& \rightarrow \int_{0}^{T} \int_{\mathbb{R} \times \mathcal{R}} F\left(\partial_{x}+\partial_{x} V \partial_{\varepsilon}\right)\left(\phi \int_{\mathcal{S}_{\varepsilon}} v_{x}(k) \chi_{\rho} d N_{\varepsilon}(k)\right) d x d \varepsilon d t
\end{aligned}
$$

as $\alpha \rightarrow 0$. Indeed, it is clear that

$$
\int_{\mathcal{S}_{\varepsilon}} v_{x}(k) \partial_{x}\left(\phi \chi_{\rho}\right) d N_{\varepsilon}(k)=\partial_{x}\left(\phi \int_{\mathcal{S}_{\varepsilon}} v_{x}(k) \chi_{\rho} d N_{\varepsilon}(k)\right) .
$$

That

$$
\int_{\mathcal{S}_{\varepsilon}} \partial_{k_{x}}\left(\phi \chi_{\rho}\right) d N_{\varepsilon}(k)=\partial_{\varepsilon}\left(\phi \int_{\mathcal{S}_{\varepsilon}} v_{x}(k) \chi_{\rho} d N_{\varepsilon}(k)\right)
$$

is easily seen in duality against a test function $\psi(\varepsilon)$ by means of the co-area formula. We note

$$
D_{\rho}(x, k)=\int_{\mathcal{S}_{\varepsilon}} v_{x}(k) \chi_{\rho} d N_{\varepsilon}(k) .
$$


We now consider the left-hand side of (4.35). We first write, using duality and the second equation (3.16),

$$
\begin{aligned}
\frac{1}{\alpha} \int_{0}^{T} & \alpha \sum_{n \in \mathbb{Z}} \int_{B} \gamma_{n \alpha}^{\text {out }}\left(f^{\alpha}\right)(k, t) \\
& \times\left(\left(I-\mathcal{B}(n \alpha)^{*}\right) \chi_{\rho}(n \alpha, k)\right) \phi(n \alpha, \varepsilon(k), t)\left|v_{x}(k)\right| d k d x d t \\
= & \int_{0}^{T} \alpha \sum_{n \in \mathbb{Z}} \int_{B} \alpha^{-1} P \gamma_{n \alpha}^{\text {out }}\left(f^{\alpha}\right)(k, t) \\
& \times\left(\left(I-\mathcal{B}(n \alpha)^{*}\right) \chi_{\rho}(n \alpha, k)\right) \phi(n \alpha, \varepsilon(k), t)\left|v_{x}(k)\right| d k d x d t
\end{aligned}
$$

We now define the piecewise constant functions $g^{\alpha, \text { out }}$ and $g^{\alpha, \text { inc }}$ by

$$
\left.\begin{array}{l}
g^{\alpha, \text { out }}(x, k, t)=\alpha^{-1} P \gamma_{n \alpha}^{\text {out }}\left(f^{\alpha}\right)(k, t) \\
g^{\alpha, \text { inc }}(x, k, t)=\alpha^{-1} P \gamma_{n \alpha}^{\text {inc }}\left(f^{\alpha}\right)(k, t)
\end{array}\right\}, \quad x \in\left[\left(n-\frac{1}{2}\right) \alpha,\left(n+\frac{1}{2}\right) \alpha\right] .
$$

From the estimates (4.4) and (4.5), the functions $\sqrt{\left|v_{x}(k)\right|} g^{\alpha, \text { out }}$ and $\sqrt{\left|v_{x}(k)\right|} g^{\alpha, \text { inc }}$ are bounded in $L^{2}([0, T] \times \mathbb{R} \times B)$. Denote by $\sqrt{\left|v_{x}(k)\right|} g^{0, \text { out }}$ and $\sqrt{\left|v_{x}(k)\right|} g^{0, \text { inc }}$ their weak limit (up to a subsequence). We note that, passing to the limit in

$$
P \gamma_{n \alpha}^{\text {inc }}\left(f^{\alpha}\right)(k, t)=\mathcal{B}(n \alpha) P \gamma_{n \alpha}^{\text {out }}\left(f^{\alpha}\right)(k, t),
$$

we have

$$
g^{0, \text { inc }}=\mathcal{B}(x) g^{0, \text { out }} .
$$

In (4.37), the factors $\left(\left(I-\mathcal{B}(n \alpha)^{*}\right) \chi_{\rho}(n \alpha, k)\right) \phi(n \alpha, \varepsilon(k), t)$ can be considered as the piecewise constant approximation of the function $\left(\left(I-\mathcal{B}(x)^{*}\right) \chi_{\rho}(x, k)\right) \phi(x, \varepsilon(k), t)$. Since this function is regular, and more precisely, uniformly continuous with respect to $x$, with values in $L^{2}(B)$, the piecewise constant approximation is an approximation in the strong topology of $L^{2}\left([0, T] \times \mathbb{R}, L^{2}(B)\right)$.

It follows that the expression (4.37) can be viewed as the $L^{2}$ dot product of a weakly converging sequence with a strongly converging one. Therefore, it converges as $\alpha \rightarrow 0$ to

$$
\int_{0}^{T} \int_{\mathbb{R} \times B} g^{0, \text { out }}(x, k, t)\left(\left(I-\mathcal{B}(x)^{*}\right) \chi_{\rho}(x, k)\right) \phi(x, \varepsilon, t)\left|v_{x}(k)\right| d k d x d t .
$$

Introducing

$$
J_{\rho}=\int_{\mathcal{S}_{\varepsilon}} g^{0, \text { out }}(x, k, t)\left(\left(I-\mathcal{B}(x)^{*}\right) \chi_{\rho}(x, k)\right)\left|v_{x}(k)\right| d N_{\varepsilon}(k),
$$

(4.38) and (4.36) yield

$$
\int_{0}^{T} \int_{\mathbb{R} \times \mathcal{R}} J_{\rho} \phi d x d \varepsilon d t=\int_{0}^{T} \int_{\mathbb{R} \times \mathcal{R}} F\left(\partial_{x}+\partial_{x} V \partial_{\varepsilon}\right)\left(\phi D_{\rho}\right) d x d \varepsilon d t
$$

which implies that

$$
J_{\rho}=-D_{\rho}\left(\partial_{x}+\partial_{x} V \partial_{\varepsilon}\right) F
$$

in $\mathcal{D}^{\prime}((0, T) \times \mathbb{R} \times \stackrel{\circ}{\mathcal{R}})$. Passing to the limit $\rho \rightarrow 0$ in (4.39) and (4.41), we shall prove that

$$
J=-D\left(\partial_{x}+\partial_{x} V \partial_{\varepsilon}\right) F
$$


Indeed, passing to the limit in (4.39) (strongly in $L^{2}$ ) and using the definition of $\chi$, we find

$$
\begin{aligned}
\lim _{\rho \rightarrow 0} J_{\rho} & =\int_{\mathcal{S}_{\varepsilon}} g^{0, \text { out }}(x, k, t)\left(\left(I-\mathcal{B}(x)^{*}\right) \chi(x, k)\right)\left|v_{x}(k)\right| d N_{\varepsilon}(k) \\
& =\int_{\mathcal{S}_{\varepsilon}} g^{0, \text { out }}(x, k, t) \frac{1}{2}\left(\left(I+\mathcal{B}(x)^{*}\right) \operatorname{sgn}\left(v_{x}\right)\right)\left|v_{x}(k)\right| d N_{\varepsilon}(k) \\
& =\int_{\mathcal{S}_{\varepsilon}} \frac{1}{2}\left((I+\mathcal{B}(x)) g^{0, \text { out }}(x, k, t)\right) v_{x}(k) d N_{\varepsilon}(k) \\
& =\int_{\mathcal{S}_{\varepsilon}} \frac{1}{2}\left(g^{0, \text { out }}+g^{0, \text { inc }}\right)(x, k, t) v_{x}(k) d N_{\varepsilon}(k) .
\end{aligned}
$$

Besides, the weak convergence of $g^{\alpha, \text { out }}, g^{\alpha, \text { inc }}$ towards $g^{0, \text { out }}, g^{0, \text { inc }}$ implies that

$$
\int_{\mathcal{S}_{\varepsilon}} \frac{1}{2}\left(g^{0, \text { out }}+g^{0, \text { inc }}\right) v_{x}(k) d N_{\varepsilon}(k)=\text { weak } \lim _{\rho \rightarrow 0} \int_{\mathcal{S}_{\varepsilon}} \frac{1}{2}\left(g^{\alpha, \text { out }}+g^{\alpha, \text { inc }}\right) v_{x}(k) d N_{\varepsilon}(k) \text {. }
$$

In view of (4.33) and of the proof of Lemma 4.5, we have

$$
\int_{\mathcal{S}_{\varepsilon}} \frac{1}{2}\left(g^{\alpha, \text { out }}+g^{\alpha, \text { inc }}\right) v_{x}(k) d N_{\varepsilon}(k)=\widetilde{J}^{\alpha},
$$

which, in (4.22), has been shown to weakly converge to $J$. Therefore,

$$
J_{\rho} \rightarrow J \quad \text { in } L^{2}((0, T) \times \mathbb{R} \times \mathcal{R}) .
$$

Let us now identify $J$ by passing to the limit in (4.41). To this aim, we use the following lemma whose proof is postponed to the end of the section.

Lemma 4.9. The diffusion constant $D(x, \varepsilon)$ is a strictly positive continuous function on $\mathbb{R} \times \stackrel{\circ}{\mathcal{R}}$.

End of the proof of Lemma 4.8. Hypothesis 4.1 implies that $D_{\rho} \rightarrow D$ as $\rho \rightarrow 0$ in $L_{\text {loc }}^{\infty}(\mathbb{R} \times \stackrel{\circ}{\mathcal{R}})$ strongly, where $D$ is given by $(2.17)$. We deduce from that and from Lemma 4.9 that for any compact set $K \in \mathbb{R} \times \stackrel{\circ}{\mathcal{R}}$, there exists a positive constant $\delta_{K}$ such that

$$
D_{\rho}(x, \varepsilon) \geq \delta_{K}, \quad \forall(x, \varepsilon) \in K,
$$

for $\rho$ small enough (depending on $K)$. This implies that $\left(\partial_{x}+\partial_{x} V \partial_{\varepsilon}\right) F$ belongs to $L_{\text {loc }}^{2}(\mathbb{R} \times \stackrel{\circ}{\mathcal{R}})$. We can therefore pass to the limit $\rho \rightarrow 0$ in $(4.41)$ and obtain the current equation (2.14) in the space $L^{2}(\mathbb{R} \times \mathcal{R})$.

Proof of Lemma 4.9. Let $\varepsilon$ be fixed in $\stackrel{\circ}{\mathcal{R}}$. We notice that we can write $(2.17)$ as $D(x, \varepsilon)=\left(\chi, \operatorname{sgn}\left(v_{x}\right)\right)_{L^{2}\left(\mathcal{S}_{\varepsilon}\right)}$. Besides, Eq. (2.18) can be recast according to

$$
\chi-\frac{1}{2} \operatorname{sgn}\left(v_{x}\right)=\mathcal{B}^{*}\left(\chi+\frac{1}{2} \operatorname{sgn}\left(v_{x}\right)\right) .
$$

We introduce $\lambda=\chi+\frac{1}{2} \operatorname{sgn}\left(v_{x}\right)$. We have $\left(I-\mathcal{B}^{*}\right) \lambda=\operatorname{sgn}\left(v_{x}\right)$ and $\left(I+\mathcal{B}^{*}\right) \lambda=2 \chi$. It follows that

$$
D=\left(\chi, \operatorname{sgn}\left(v_{x}\right)\right)_{L^{2}\left(\mathcal{S}_{\varepsilon}\right)}=\frac{1}{2}\left(\left(I+\mathcal{B}^{*}\right) \lambda,\left(I-\mathcal{B}^{*}\right) \lambda\right)_{L^{2}\left(\mathcal{S}_{\varepsilon}\right)}=\frac{1}{2}\left(|\lambda|^{2}-\left|\mathcal{B}^{*} \lambda\right|^{2}\right) \geq 0 .
$$


Furthermore, $D$ can be zero only if $\lambda=\chi+\frac{1}{2} \operatorname{sgn}\left(v_{x}\right)=C(\varepsilon(k))$. But the condition $(\chi, 1)_{L^{2}\left(\mathcal{S}_{\varepsilon}\right)}=0$ implies that $C=0$ and $\chi=-\frac{1}{2} \operatorname{sgn}\left(v_{x}\right)$. But then $\chi$ cannot be a solution of (2.18). Therefore, the inequality is strict, which yields the positivity of $D$.

The continuity of $D$ is a consequence of that of $D_{\rho}$ (which follows from Hypothesis $4.1)$, and of the convergence of $D_{\rho}$ to $D$ in $L_{\text {loc }}^{\infty}(\mathbb{R} \times \stackrel{\circ}{\mathcal{R}})$.

\section{Examples.}

5.1. Isotropic reflection-transmission. This case corresponds to the choice

$$
\sigma\left(x, k^{\prime}, k\right)=\frac{2}{\mathcal{V}(\varepsilon)} \begin{cases}t(x, \varepsilon), & \text { if }\left(v_{x}\left(k^{\prime}\right) \geq 0 \text { and } v_{x}(k) \geq 0\right) \\ 1-t(x, \varepsilon), & \text { if }\left(v_{x}\left(k^{\prime}\right) \geq 0 \text { and } v_{x}(k) \leq 0\right) \\ & \text { or }\left(v_{x}\left(k^{\prime}\right) \leq 0 \text { and } v_{x}(k) \geq 0\right),\end{cases}
$$

where $t(x, \varepsilon) \in[0,1]$ is the transmission probability of particles of energy $\varepsilon$ through the interface, $1-t(x, \varepsilon)$ is the reflection probability and $\mathcal{V}(\varepsilon)$ is the normalizing factor (3.13). In this case, it is easy to see that $\mathcal{B}$ is selfadjoint. After some straightforward computations, one gets

$$
\chi(x, k)=\frac{t}{2(1-t)} \operatorname{sgn}\left(v_{x}\right), \quad D=\frac{t}{2(1-t)} \mathcal{V} .
$$

Hypothesis 3.2 on $\mathcal{B}$ require that $0<t<1$. If furthermore, $t$ is continuous with respect to $(x, \varepsilon)$ it is clear that $\chi$ satisfies Hypothesis 4.1 .

5.2. Clean interface: parallel momentum preserved. This case is opposite to the preceding one. We suppose that the interface is perfectly clean, so that momentum in the direction parallel to the interface is preserved. In this case, only a one-dimensional momentum space is considered: the component of momentum $k_{x}$ in the normal direction to the interface. It is supposed that the directional energy $\varepsilon_{x}\left(k_{x}\right)$ in this direction can be defined (this is possible in the case of a parabolic band structure, but is only an approximation in the general case), such that the velocity in this direction is written $v_{x}=\partial_{k_{x}} \varepsilon_{x}\left(k_{x}\right)$. The transmission probability becomes a function of $\varepsilon_{x}\left(k_{x}\right)$ only. The limit model is a SHE model of the form (2.13), (2.14) with

$$
\begin{aligned}
N(\varepsilon) & =\sum_{k_{x} \text { s.t. } \varepsilon_{x}\left(k_{x}\right)=\varepsilon} \frac{2}{\left|v_{x}\left(k_{x}\right)\right|}, \\
\chi & =\frac{t}{2(1-t)} \operatorname{sgn}\left(v_{x}\right), \\
D & =\sum_{k_{x} \text { s.t. } \varepsilon_{x}\left(k_{x}\right)=\varepsilon} \frac{t}{2(1-t)}
\end{aligned}
$$

With the same hypotheses on $t$ as in the previous case, we can easily show that $\chi$ satisfies Hypothesis 4.1. 


\section{REFERENCES}

[1] R. Alexandre and K. Hamdache, Homogenization of kinetic equations in nonhomogeneous medium, preprint

[2] G. Allaire, Homogenization and two scale convergence, SIAM Math. Anal. 23, 1482-1518 (1992)

[3] G. Allaire and G. Bal, Homogenization of the criticality spectral equation in neutron transport, Math. Model Numer. Anal. 33, 721-746 (1999)

[4] C. Bardos, Problèmes aux limites pour les équations aux dérivées partielles du premier ordre à coefficients réels; Théorèmes d'approximation; application à l'équation de transport, Ann. Scient. École Norm. Sup. 4, 185-233 (1970)

[5] H. Babovsky, C. Bardos, and T. Platkowski, Diffusion approximation for a Knudsen gas in a thin domain with accommodation on the boundary, Asymptotic Analysis 3, 265-289, (1991)

[6] C. Bardos, F. Golse, and B. Perthame, The Rosseland approximation for the radiative transfer equations, Comm. Pure Appl. Math. 40, 691-721 (1987) and 42, 891-894 (1989)

[7] C. Bardos, R. Santos, and R. Sentis, Diffusion approximation and computation of the critical size, Trans. Amer. Math. Soc. 284, 617-649 (1984)

[8] N. Ben Abdallah and P. Degond, On a hierarchy of macroscopic models for semiconductors, J. Math. Phys. 37, 3306-3333 (1996)

[9] A. Bensoussan, J. L. Lions, and G. Papanicolaou, Boundary layers and homogenization of transport processes, Publ. RIMS Kyoto Univ. 15, 53-157 (1979)

[10] H. Brézis, Analyse Fonctionnelle, théorie et applications, Masson, Paris, 1983

[11] C. Cercignani, The Boltzmann equation and its applications, Springer-Verlag, New York, 1998

[12] P. Degond, Mathematical modelling of microelectronics semiconductor devices. Some current topics on nonlinear conservation laws, AMS/IP Studies in Advanced Mathematics, vol. 15, Amer. Math. Soc. and International Press, 2000, pp. 77-110

[13] P. Degond, A model of near-wall conductivity and its application to plasma thrusters, SIAM J. Appl. Math. 58, 1138-1162 (1998)

[14] P. Degond and S. Mancini, Diffusion driven by collisions with the boundary, Asymptotic Anal. 27, 47-73 (2001)

[15] P. Degond and K. Zhang, Diffusion approximation of a scattering matrix model of semiconductor superlattices, preprint, 1999

[16] P. Dmitruk, A. Saul, and L. Reyna, High electric field approximation in semiconductor devices, Appl. Math. Letters 5, 99-102, (1992)

[17] W. E, Homogenization of linear and nonlinear transport equations, Comm. Pure Appl. Math. 45, 301-326 (1992)

[18] L. Esaki and R. Tsu, Superlattice and negative differential conductivity in semiconductors, IBM J. Res. Develop. 14, 61 (1970)

[19] H. Federer, Geometric Measure Theory, Springer-Verlag, Berlin, 1969

[20] E. Frenod and K. Hamdache, Homogenisation of transport kinetic equations with oscillating potentials, Proc. Roy. Soc. Edinburgh Sect. A 126, 1247-1275 (1996)

[21] V. Girault and P. A. Raviart, Finite element methods for the Navier-Stokes equations, SpringerVerlag, Berlin, 1986

[22] N. Goldsman, L. Henrickson, and J. Frey, A physics based analytical numerical solution to the Boltzmann transport equation for use in device simulation, Solid State Electron. 34, 389-396 (1991)

[23] F. Golse and F. Poupaud, Limite fluide des équations de Boltzmann des semiconducteurs pour une statistique de Fermi-Dirac, Asymptotic Analysis 6, 135-160 (1992)

[24] T. Goudon and F. Poupaud, Approximation by homogenization and diffusion of kinetic equations, Comm. Partial Differential Equations 26, 537-569 (2001)

[25] H. T. Grahn (ed.), Semiconductor Superlattices, growth and electronic properties, World Scientific, Singapore, 1995

[26] E. W. Larsen, Neutron transport and diffusion in inhomogeneous media I, J. Math. Phys. 16, 1421-1427 (1975)

[27] E. W. Larsen, Neutron transport and diffusion in inhomogeneous media II, Nuclear Sci. Engrg. 60, 357-368 (1976)

[28] E. W. Larsen and J. B. Keller, Asymptotic solution of neutron transport problems for small mean free paths, J. Math. Phys. 15, 75-81 (1974) 
[29] G. Nguetseng, A general convergence result for a functional related to the theory of homogenization, SIAM Math. Anal. 20, 608-623 (1989)

[30] A. Pazy, Semigroups of linear operators and applications to partial differential equations, SpringerVerlag, New York, 1983

[31] F. Poupaud, Diffusion approximation of the linear semiconductor equation: Analysis of boundary layers, Asymptotic Analysis 4, 293-317 (1991)

[32] F. Poupaud, Étude de l'opérateur de transport $A u=a \nabla u$, manuscript, unpublished

[33] D. Ventura, A. Gnudi, G. Baccarani, and F. Odeh, Multidimensional spherical harmonics expansion of Boltzmann equation for transport in semiconductors, Appl. Math. Letters 5, 85-90 (1992)

[34] C. Weisbuch and B. Vinter, Quantum Semiconductor Structures, fundamentals and applications, Academic Press, Boston, 1991 